\title{
Biology-inspired robust dive plane control of non-linear AUV using pectoral-like fins
}

\author{
Subramanian Ramasamy and Sahjendra N. Singh* \\ Department of Electrical and Computer Engineering, University of Nevada Las Vegas Las Vegas, NV, USA
}

(Received 12 March 2009; final version received 4 February 2010)

\begin{abstract}
The development of a control system for the dive plane control of non-linear biorobotic autonomous underwater vehicles, equipped with pectoral-like fins, is the subject of this paper. Marine animals use pectoral fins for swimming smoothly. The fins are assumed to be oscillating with a combined pitch and heave motion and therefore produce unsteady control forces. The objective is to control the depth of the vehicle. The mean angle of pitch motion of the fin is used as a control variable. A computational-fluid-dynamics-based parameterisation of the fin forces is used for control system design. A robust servo regulator for the control of the depth of the vehicle, based on the non-linear internal model principle, is derived. For the control law derivation, an exosystem of third order is introduced, and the non-linear time-varying biorobotic autonomous underwater vehicle model, including the fin forces, is represented as a non-linear autonomous system in an extended state space. The control system includes the internal model of a $k$-fold exosystem, where $k$ is a positive integer chosen by the designer. It is shown that in the closed-loop system, all the harmonic components of order up to $k$ of the tracking error are suppressed. Simulation results are presented which show that the servo regulator accomplishes accurate depth control despite uncertainties in the model parameters.
\end{abstract}

Keywords: pectoral fin control system; non-linear biorobotic autonomous underwater vehicle control; robust output regulation; non-linear servo regulation; non-linear internal model principle

\section{Introduction}

Aquatic animals swim smoothly through water using a variety of- oscillating fins, and birds and insects fly using flapping wings (Azuma 1992; Luca 1999; Sfakiotakis et al. 1999; Fish 2004; Lauder and Drucker 2004; Kato and Kamimura 2008) . The extraordinary maneuverability of marine animals, birds and insects is the result of their ability to generate and control large forces from unsteady hydrodynamics and aerodynamics, respectively. Presently, researchers are involved in developing biorobotic autonomous underwater vehicles (BAUVs) which have the ability to swim like marine animals (Bandyopadhyay 2005). In literature, mulitiple oscillating fins mounted on AUVs have been proposed to generate control forces for propulsion and manoeuvering (Triantafyllou and Triantafyllou 1995; Kato 2000, 2002; Triantafyllou et al. 2004; Bandyopadhyay et al. 2008). A robotic turtle-like underwater vehicle, a robotic bat and a flying insect have been also developed by researchers (Luca 1999; Seo et al. 2008; Chung et al. 2009). Laboratory experiments have been performed to obtain fin forces of oscillating fins (Triantafyllou and Triantafyllou 1995; Triantafyllou et al. 2003, 2004; Bandyopadhyay 2008). Computational fluid dynamics (CFD) methods have been also used to derive the fin forces (Singh et al. 2004; Narasimhan et al. 2006). The unsteady forces are complex periodic functions of the oscillation parameters (bias angle, amplitude, frequency of oscillation, relative phase angle, etc.). The mathematical models of BAUVs, including the fin forces, are non-linear and time-varying.

In literature, methods of averaging and discretisation of time-periodic systems have been proposed for the control of BAUVs. By averaging method, one obtains an approximate average time-invariant representation of the BAUV model for simplicity in control law design (Luca 1999). But the control system designed based on the time-invariant average model ignores the effect of time-varying fin forces on the vehicle motion. As such in the closed-loop system, the tracking error responses exhibit fluctuations caused by the harmonic components of the fin forces in the steady state. Based on exact discrete-time models of BAUVs (Singh et al. 2004; Narasimhan et al. 2006), sampled-data control systems have been developed. For BAUV models with parametric uncertainties, discrete-time adaptive laws have been also designed (Naik and Singh 2007; Naik et al. 2009). Of course, exact discretisation is not possible for non-linear models of BAUVs. Moreover, discrete-time controllers can give zero tracking error only at the sampling instants, and in the closed-loop system, large inter-sample excursions may exist. Besides these approaches, fuzzy and neural control of BAUVs have been considered (Yamamoto 1995; Kato 2000, 2002). Open-loop control of a BAUV equipped with

*Corresponding author. Email: sahaj@ee.unlv.edu

ISSN: $1176-2322$ print / 1754-2103 online

Copyright (C) 2010 Taylor \& Francis

DOI: $10.1080 / 11762321003760936$

http://www.informaworld.com 
six oscillating fins using a cluster of inferior olive neurons has been also attempted (Bandyopadhyay et al. 2008).

In this paper, a new approach for the dive plane control of a non-linear AUV equipped with oscillating pectoral-like fins is presented. The method is based on the non-linear servo regulation theory (Huang 1995, 2004). The objective is to control the depth of the BAUV. The fins are assumed to oscillate harmonically and have a combined pitch and heave motion. The pitch bias angle of the fin is treated as a control input. Oscillating fins produce time-periodic unsteady control forces. A CFD-based parameterisation of the fin forces is used for the design of control system. An exosystem of third order is introduced to model the periodic forces, and the time-varying non-linear model of the BAUV is represented as an autonomous non-linear system in an extended state space. For the depth control, based on the non-linear servo regulation theory, an internal model of $k$-fold exosystem driven by the tracking error is constructed, where $k$ is a positive integer chosen to give desirable tracking accuracy. The $k$-fold exosystem has ability to produce monomials of degree up to $k$ of the state variables of the exosystem. Then the composite system including the linearised model of the BAUV and the internal model of the $k$-fold exosystem is stabilised to obtain a robust state feedback control law for the depth control. It is shown that the controller, including the internal model in the loop, suppresses harmonic fluctuations of degree up to $k$ in the tracking error responses. This desirable closed-loop property is not possible using the method of averaging (Luca 1999) or discretisation (Singh et al. 2004; Narasimhan et al. 2006; Naik and Singh 2007). Simulation results are presented which show that the servo regulator accomplishes set point control of the depth precisely in spite of large parameter uncertainties in fin forces.

\section{Autonomous underwater vehicle model and control problem}

Figure 1 shows the schematic of a typical BAUV. Two fins resembling the pectoral fins of fish are symmetrically attached to the vehicle. The vehicle moves in the dive plane $\left(X_{I}-Z_{I}\right.$ plane), where $O_{I} X_{I} Z_{I}$ is an inertial coordinate system. $O_{B} X_{B} Z_{B}$ is body-fixed coordinate system with its origin at the centre of buoyancy. $X_{B}$ is in the forward direction, and $Z_{B}$ points down. Each fin has two degrees of freedom (pitch and heave) and oscillates harmonically. We assume that the combined pitch-heave motion of the fin is described as follows:

$$
\begin{aligned}
h(t) & =h_{m} \sin \left(\omega_{f} t\right), \\
\psi(t) & =\beta+\psi_{m} \sin \left(\omega_{f} t+v_{1}\right),
\end{aligned}
$$

where $h$ and $\psi$ correspond to heave and pitch angle of the fin, $h_{m}$ and $\psi_{m}$ are the amplitudes of linear and angular oscillations, $\beta$ is the pitch bias angle, $\omega_{f}$ (in radians per

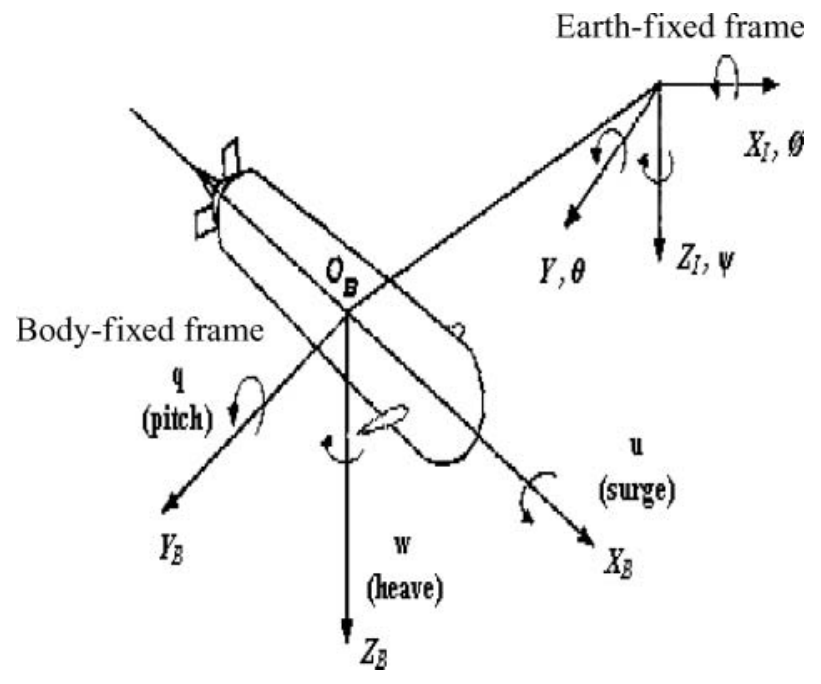

Figure 1. Model of BAUV.

second) is the frequency of oscillations of fins and $v_{1}$ is the phase difference between the pitching and heaving motion.

A CFD-based parameterisation of the fin force and moment is used in this paper. (Readers may find the parameterised fin force and moment in the paper by Narasimhan et al. 2006.) For simulating the flow past oscillating foils, a finite-difference-based, Cartesian grid immersed boundary solver was used (Nazzar et al. 2003). The Eulerian form of the incompressible Navier-Stokes equations was discretised on a Cartesian mesh (Udaykumar et al. 2001). For including the effect of the small sub-grid flow scales on the large resolved scales, the large-eddy-simulation approach was used to develop the code. A Lagrangian dynamic model (Meneveau et al. 1996) was used to estimate the sub-gridscale eddy viscosity. The details of the numerical method and validation of the code can be found in Bozcurttas et al. (2005).

Based on the numerical results obtained by the CFD code, the lift component $\left(f_{p}\right)$ and pitching moment $\left(m_{p}\right)$ generated by the oscillating fin can be described by the Fourier series given by (Narasimhan et al. 2006)

$$
\begin{aligned}
f_{p}(t) & =\sum_{n=0}^{M}\left[f_{n}^{s}(\beta) \sin \left(n \omega_{f} t\right)+f_{n}^{c}(\beta) \cos \left(n \omega_{f} t\right)\right], \\
m_{p}(t) & =\sum_{n=0}^{M}\left[m_{n}^{s}(\beta) \sin \left(n \omega_{f} t\right)+f_{n}^{c}(\beta) \cos \left(n \omega_{f} t\right)\right],
\end{aligned}
$$

where $f_{n}^{a}(\beta)$ and $m_{n}^{b}(\beta), a \in\{s, c\}$, are the Fourier coefficients, and $M$ is an integer such that the neglected harmonics have insignificant effect.

The Fourier coefficients are non-linear functions of the pitch bias angle. The net lateral force because of two fins is given by $f_{p v}=-2 f_{p}$ and $m_{p v}=2\left(d_{c g f} \cdot f_{p}+m_{p}\right)$, 
respectively, where $d_{c g f}$ is the moment arm because of fin location.

We assume that vehicle's forward speed $u$ is held constant by some control mechanism. The equations of motion of a neutrally buoyant vehicle are described by (Fossen 1994)

$$
\begin{aligned}
& m\left(\dot{w}-u q-z_{G} \dot{q}^{2}-x_{G} \dot{q}\right) \\
& \quad=0.5 \rho l^{4} z_{\dot{q}}^{\prime} \dot{q}+0.5 \rho l^{3}\left(z_{w}^{\prime} \dot{w}+z_{q}^{\prime} q u\right) \\
& \quad+0.5 \rho l^{2} z_{w}^{\prime} w u+f_{p v}, \\
& I_{y} \dot{q}+m z_{G}(\dot{u}+w q)-m x_{G}(\dot{w}-u q) \\
& \quad=0.5 \rho l^{5} M_{\dot{q}}^{\prime} \dot{q}+0.5 \rho l^{4}\left(M_{\dot{w}}^{\prime}+M_{q}^{\prime} q u\right) \\
& \quad+0.5 \rho l^{3} M_{w}^{\prime} w u-x_{G B} W \cos \theta-z_{G B} W \sin \theta+m_{p v}, \\
& \dot{z}=-u \sin (\theta)+w \cos (\theta),
\end{aligned}
$$

where $I_{y}$ is the moment of inertia, $m$ is the mass, $w$ is the heave velocity, $\theta$ is the pitch angle, $q=\dot{\theta}, x_{G B}=x_{G}-x_{B}$, $z_{G B}=z_{G}-z_{B}, l=$ body length, $\rho=$ density and $z$ is the depth. $f_{p v}$ and $m_{p v}$ are the net force and moment acting on the vehicle because of pectoral fins. The primed variables $\left(M_{\dot{q}}^{\prime}, z_{\dot{q}}^{\prime}, M_{\dot{w}}^{\prime}\right.$, etc.) are the non-dimensionalised hydrodynamic coefficients. Here $\left(\left(x_{B}, z_{B}\right)=0\right)$ and $\left(x_{G}, z_{G}\right)$ denote the coordinates of the centre of buoyancy and centre of gravity (cg) respectively.

Let $z_{r}=z^{*}$ be a constant reference signal. Defining the state vector $x=\left(x_{1}, x_{2}, x_{3}, x_{4}\right)^{T}=(w, q, z, \theta)^{T} \in R^{4}$, solving (4) and substituting for $f_{p v}$ and $m_{p v}$ from (3) gives the state variable representation of the BAUV of the form

$$
\begin{aligned}
& \dot{x}=A x+B_{1}\left[\begin{array}{c}
f_{p} \\
m_{p}
\end{array}\right]+n_{l}(x), \\
& e=z-z_{r},
\end{aligned}
$$

where $e$ is the tracking error and $n_{l}(x)$ denotes the vector because of the non-linear functions of (4). In view of (3), the state Equation (5) is a non-linear time-varying system.

We are interested in representing (5) as a time-invariant system. For this, we select an exosystem

$$
\left(\begin{array}{c}
\dot{v}_{0} \\
\dot{v}_{1} \\
\dot{v}_{2}
\end{array}\right)=\left(\begin{array}{ccc}
0 & 0 & 0 \\
0 & 0 & -\omega_{f} \\
0 & \omega_{f} & 0
\end{array}\right)\left(\begin{array}{c}
v_{0} \\
v_{1} \\
v_{2}
\end{array}\right)=A_{v} v
$$

where $v=\left(v_{0}, v_{1}, v_{2}\right)^{T} \in R^{3}$. Define $v_{p}=\left(v_{1}, v_{2}\right)^{T}$. Using (6), one can generate any constant and sinusoidal signals $\sin \left(n \omega_{f} t\right)$ and $\cos \left(n \omega_{f} t\right)$ for any integer $n$. This can be verified easily. Let $v_{0}=1, v_{1}=\cos \omega_{f} t$ and $v_{2}=\sin \omega_{f} t$, then $v_{1}$ and $v_{2}$ satisfy (6), and one can easily show that

$$
\begin{aligned}
\sin 2 \omega_{f} t & =2 v_{1} v_{2}, \\
\cos 2 \omega_{f} t & =\left(v_{1}^{2}-v_{2}^{2}\right),
\end{aligned}
$$

$$
\begin{aligned}
& \sin 3 \omega_{f} t=2 v_{1}^{2} v_{2}+\left(v_{1}^{2}-v_{2}^{2}\right) v_{2}, \\
& \cos 3 \omega_{f} t=\left(v_{1}^{2}-v_{2}^{2}\right) v_{1}-2 v_{1} v_{2}^{2} .
\end{aligned}
$$

Continuing this process, one can easily show that $\sin \left(n \omega_{f} t\right)$ and $\cos \left(n \omega_{f} t\right)$ can be expressed as homogeneous polynomials, whose each term is monomial in variable $v_{1}$ and $v_{2}$ of degree $n$. As such one can express the fin force and moment as functions of state vector $v$ in the form

$$
\begin{aligned}
\left(\begin{array}{c}
f_{p}(t, \beta) \\
m_{p}(t, \beta)
\end{array}\right) & =\gamma_{0}(\beta)+\sum_{n=1}^{M} \gamma_{n}^{s}(\beta) \pi_{n}^{s}\left(v_{p}\right)+\gamma_{n}^{c}(\beta) \pi_{n}^{c}\left(v_{p}\right) \\
& =g_{f}(v, \beta)
\end{aligned}
$$

and $\pi_{n}^{s}\left(v_{p}\right)=\sin n \omega_{f} t$ and $\pi_{n}^{c}\left(v_{p}\right)=\cos n \omega_{f} t$ are homogeneous polynomials in variables $v_{1}$ and $v_{2}$ of degree $n$, and

$$
\begin{aligned}
& \gamma_{0}(\beta)=\left(\begin{array}{c}
f_{0}^{c}(\beta) \\
m_{0}^{c}(\beta)
\end{array}\right), \\
& \gamma_{n}^{s}(\beta)=\left(\begin{array}{c}
f_{n}^{s}(\beta) \\
m_{n}^{s}(\beta)
\end{array}\right), \\
& \gamma_{n}^{c}(\beta)=\left(\begin{array}{c}
f_{n}^{c}(\beta) \\
m_{n}^{c}(\beta)
\end{array}\right) .
\end{aligned}
$$

Using (8) in (5) gives a time-invariant representation of (5) of the form

$$
\begin{aligned}
\dot{x} & =A(p) x+g\left(v_{p}, \beta, p\right)+n_{l}(x, p) \triangleq g_{x}(x, v, p), \\
e & =x_{3}-z^{*} v_{0},
\end{aligned}
$$

where $g(v, \beta, p)=B_{1} g_{f}(v, \beta)$ and $p=R^{m}$ denotes the vector consisting of all the unknown parameters of the BAUV model including fin forces. For example, $p$ includes the uncertain parameters of the Fourier coefficients.

Expanding the non-linear terms of (9) in Taylor series, one can represent (9) in the form

$$
\begin{aligned}
& \dot{x}=A(p) x+B(p) u_{c}+E(p) v+n_{l 2}\left(u_{c}, v, x, p\right), \\
& e \triangleq H_{1} x+H_{2} v \triangleq h\left(x, v_{0}\right),
\end{aligned}
$$

where $u_{c}=\beta, H_{1}=(0,0,1,0), H_{2}=\left(-z^{*}, 0,0\right), n_{l 2}$ denotes non-linear vector functions of second- and higherorder terms in $\beta, v_{1}, v_{2}$ and $x$, and

$$
\begin{aligned}
& B(p)=\frac{\partial g}{\partial \beta}(0,0, p), \\
& E(p)=\left[g(0,0, p), \frac{\partial g}{\partial v_{p}}(0,0, p)\right] .
\end{aligned}
$$

Note that $E_{1} v_{0}\left(v_{0}=1\right)$ is a constant vector and $E_{1}$ is the first column of $E$. For the choice of $v_{0}=1, v_{1}=\cos \omega_{f} t$, $v_{2}=\sin \omega_{f} t$, the system (5) and (10) are equivalent. 
For the purpose of control law derivation, we embed the system (5) in a larger class of system (10) in which we allow $v \in V$, an open set in $R^{3}$. Of course, unknown coefficients of two sinusoids $\sin \left(\omega_{f} t\right)$ and $\cos \left(\omega_{f} t\right)$ can be merged with $v_{p}$ and remaining unknown parameters are elements of $p$. We have set the goal for approximate tracking for practical reasons. It will be seen that the design of control law such that $e$ tends to zero is a difficult problem because of timevarying periodic fin forces. The class of control laws of interest is of the form $u_{c}=k_{c}\left(x, x_{s}\right)$, where $x_{s} \in R^{n_{c}}$ is state vector of a dynamic system

$$
\dot{x}_{s}=g_{s}\left(x_{s}, e\right)
$$

for an appropriate choice of vector functions $g_{s}\left(x_{s}, e\right)$. We observe that the tracking error is an input signal to the dynamical system (11).

Define $x_{c}=\left(x^{T}, x_{s}^{T}\right) \in R^{4+n_{c}}$. Then the closed-loop system can be written as

$$
\begin{aligned}
\dot{x}_{c} & =\left[\begin{array}{c}
A(p) x+B k_{c}\left(x, x_{s}\right)+E(p) v \\
+n_{l 2}\left(k_{c}\left(x, x_{s}\right), v, x, p\right) \\
g_{s}\left(x_{s}, h\left(x, v_{0}\right)\right)
\end{array}\right] \\
& \triangleq g_{c}\left(x_{c}, v, p\right) .
\end{aligned}
$$

Let the nominal value of the unknown parameter vector $p$ be $p^{*}$ and $\tilde{p}=p-p^{*}$ be the perturbation from the nominal value. We assume that $\tilde{p} \in \Omega_{p}$, an open set surrounding $\tilde{p}=$ 0 . We introduce the following definition to be used later.

Definition. Let $V$ be an open neighborhood of the origin $R^{3}$. A sufficiently smooth function $o^{k}: V \rightarrow R$ is said to be zero up to the $k$ th order if $o^{k}(0)=0$ and its all partial derivatives of order less than or equal to $k$ vanish at $v=0$.

We are interested in the design of a $k$ th-order non-linear robust control system (termed $k$ th-order servo regulator) such that the closed-loop system (12) has the following properties.

Property 1: All the eigenvalues of the matrix $\frac{\partial g_{c}}{\partial x_{c}}\left(0,0, p^{*}\right)$ have negative real parts.

Property 2: For all sufficiently small $x_{c}(0), v(0)$ and $\tilde{p}$, the trajectory $\left(x_{c}(t), v(t)\right)$ of the composite system (12) and (6) satisfies

$$
\begin{aligned}
& \lim _{t \rightarrow \infty}\left(e(t)-o^{k}(v(t))\right. \\
& \quad=\lim _{t \rightarrow \infty}\left(h(x(t), v(t))-o^{k}(v(t))=0,\right.
\end{aligned}
$$

where $k$ is the chosen positive integer.

The Property 2 implies that steady-state tracking error of the closed-loop system is zero up to $k$ th order. By choosing $k$ large enough, designer can accomplish desired tracking error accuracy in the steady state.

\section{Control law}

In this section, the question of existence of a solution of the posed $k$ th-order output regulation is considered. Based on the work of Huang $(1995,2004)$, the following result is stated.

Theorem 1. Suppose that in the closed-loop system (12), Property 1 holds. Then the closed-loop system also satisfies Property 2 if and only if there exists sufficiently smooth functions $X_{c}(v, p)=\left[X^{T}(v, p), X_{s}^{T}(v, p)\right]^{T}$ with $X_{c}\left(0, p^{*}\right)=0$ which satisfies for $v \in V$ and $\tilde{p} \in \tilde{\Omega}_{p}$

$$
\begin{aligned}
\frac{\partial X_{c}(v, p)}{\partial v} A_{v} v & =g_{c}\left(X_{c}(v, p), v, p\right), \\
\mathbf{e}(v, p) & =\mathbf{h}(X(v, p), v)=o^{k}(v) .
\end{aligned}
$$

It is possible to synthesise a control law to solve the problem of the $k t h$-order regulation using the solution of (14). However, it is not easy to solve the partial differential Equation (14), and moreover, the solution depends on the unknown parameter $p$.

\subsection{Internal model}

In this sub-section, we now seek a solution for the regulation problem based on the non-linear internal model principle (Huang 2004). This approach avoids the computation of $X_{c}(v, p)$. To motivate the construction of an internal model, we look into an approximate solution of (14). One can attempt to obtain a solution of (14) by selecting $X_{c}(v, p)=\left(X^{T}(v, p), X_{s}^{T}(v, p)\right)^{T}$ and $U_{c}(v, p)=$ $k_{c}\left(X(v, p), X_{s}(v, p)\right)$ as polynomial functions of variables $v_{0}, v_{1}$ and $v_{2}$ given by

$$
\begin{aligned}
& X(v, p)=\sum_{l=1}^{k} X_{l p} v^{[l]}+o^{k}(v), \\
& X_{s}(v, p)=\sum_{l=1}^{k} X_{s_{l p}} v^{[l]}+o^{k}(v), \\
& U_{c}(v, p)=\sum_{l=1}^{k} U_{c l p} v^{[l]}+o^{k}(v),
\end{aligned}
$$

where $v^{[1]}=\left[v_{0}, v_{1}, v_{2}\right]^{T}$ and $v^{[l]}=v_{p}^{[l]}=\left[v_{1}^{l}, \quad v_{1}^{l-1}\right.$ $\left.v_{2}, v_{1}^{l-2} v_{2}^{2}, \ldots, v_{2}^{l}\right]^{T}$, for $l=2,3, \ldots$, and

$$
e(v, p)=\mathbf{h}(X(v, p), v)=\sum_{l=1}^{k} Y_{l p} v^{[l]}+o^{k}(v) .
$$


Here $X_{l p}, X_{s l p}, U_{c l p}$ and $Y_{l p}$ are constant matrices of appropriate dimensions depending, perhaps, on $p$. Each component of the vector polynomial $v_{p}^{[l]}$ is monomial in variables $v_{1}$ and $v_{2}$ of degree $l$. Essentially the elements of $v_{p}^{[l]}$ form a basis for homogeneous polynomials of degree $l$ in variables $v_{1}$ and $v_{2}$. In view of (15), to satisfy (13) it is essential to design a control law which can cancel all the terms of $v^{[l]}, l=1, \ldots, k$, occurring in $e(v, p)=\mathbf{h}(X(v, p), v)$ in steady state. This requires construction of a dynamic system (termed internal model of $k$-fold exosystem) which can produce signals $v^{[l]}, l=1, \ldots, k$. The $k$-fold exosystem can be constructed as follows. First of all, $v^{[1]}=\left(v_{0}, v_{1}, v_{2}\right)^{T}$ and $v_{p}^{[l]}$ satisfy

$$
\begin{aligned}
& \dot{v}^{[1]}=A_{v} v^{[1]} \triangleq A^{[1]} v^{[1]}, \\
& \dot{v}_{p}^{[l]}=A_{p}^{[l]} v_{p}^{[l]}, l=2,3, \ldots, k,
\end{aligned}
$$

where

$$
A_{p}=\left[\begin{array}{ll}
0 & -\omega_{f} \\
\omega_{f} & 0
\end{array}\right]
$$

and $A_{p}^{[j]}$ are appropriate matrices. (The expressions for $A_{p}^{[l]}$ and their characteristic polynomials, for $l=1, \ldots, 4$, are collected in the appendix.)

Define a state vector

$$
v_{k f}=\left[\begin{array}{c}
v^{[1]} \\
\cdot \\
\cdot \\
v_{p}^{[k]}
\end{array}\right] .
$$

The vector $v_{k f}$ satisfies the differential equation

$$
\begin{aligned}
\dot{v}_{k f} & =\operatorname{diag}\left[A^{[1]}, A_{p}^{[2]}, \ldots, A_{p}^{[k]}\right] v_{k f} \\
& \triangleq A_{k f} v_{k f},
\end{aligned}
$$

where $A_{k f}=\operatorname{diag}\left(A^{[1]}, A_{p}^{[2]}, \ldots, A_{p}^{[k]}\right)$. The system (20) is the $k$-fold exosystem which generates not only the exogenous signal $v$, but also the higher-order terms of the exogenous signal $v_{p}$ up to order $k$. According to the internal model principle, for $k$ th-order robust regulator design (Huang 2004), one introduces an internal model of $k$-fold exosystem (19).

The roots of the minimum polynomial of $A_{k f}$ are precisely given by all the distinct members of the following set:

$$
\begin{aligned}
\Lambda_{k} & =\left\{\lambda \mid \lambda=0 \text { and } j \omega_{f}\left(l_{1}-l_{2}\right) ;\right. \\
l_{1}+l_{2} & \left.=l ; l_{1}, l_{2}=0,1, \ldots, l ; l=1,2, \ldots, k\right\} .
\end{aligned}
$$

Now the internal model is constructed using the minimum polynomial of $A_{k f}$ of the form

$$
\dot{x}_{s}=G_{1} x_{s}+G_{2} e,
$$

where $x_{s} \in R^{c}$ and

$$
\begin{aligned}
G_{1}= & \operatorname{diag}\left(0,\left[\begin{array}{cc}
0 & -\omega_{f} \\
\omega_{f} & 0
\end{array}\right],\left[\begin{array}{cc}
0 & -2 \omega_{f} \\
2 \omega_{f} & 0
\end{array}\right], \ldots,\right. \\
& {\left.\left[\begin{array}{cc}
0 & -k \omega_{f} \\
k \omega_{f} & 0
\end{array}\right]\right) . }
\end{aligned}
$$

The vector $G_{2}$ is chosen such that the pair $\left(G_{1}, G_{2}\right)$ is controllable. It can be verified that $G_{2}$ is given by

$$
G_{2}=\left[\begin{array}{c}
b_{0} \\
0 \\
b_{1} \\
0 \\
b_{2} \\
\cdot \\
\cdot \\
\cdot \\
\cdot \\
0 \\
b_{k}
\end{array}\right]
$$

which satisfies the controllability property of pair $\left(G_{1}, G_{2}\right)$ as long as $b_{i} \neq 0, i=0, \ldots, k$.

\subsection{Stabiliser design}

For completing the design, all one now has to do is to stabilise the closed-loop system. For this purpose, consider the augmented system (10) and (21) given by

$$
\begin{aligned}
\frac{d}{d t}\left[\begin{array}{c}
x \\
x_{s}
\end{array}\right]= & {\left[\begin{array}{cc}
A(p) & 0 \\
G_{2} H_{1} & G_{1}
\end{array}\right]\left(\begin{array}{c}
x \\
x_{s}
\end{array}\right)+\left[\begin{array}{c}
B(p) \\
0
\end{array}\right] u_{c} } \\
& +\left[\begin{array}{c}
n_{l 2}\left(u_{c}, x, v, p\right) \\
G_{2} H_{2} v
\end{array}\right]+\left[\begin{array}{c}
E(p) \\
0
\end{array}\right] v .
\end{aligned}
$$

Now one needs to find a control law such that $(x=0$, $x_{s}=0$ ) of the non-linear system (24) is exponentially stable for $v=0$. For exponential stabilization of the origin, it is sufficient to stabilise the linearised model obtained from (24), which for $v=0$, is given by

$$
\begin{aligned}
\frac{d x_{c}}{d t} & =\left[\begin{array}{cc}
A(p) & 0 \\
G_{2} H_{1} & G_{1}
\end{array}\right] x_{c}+\left[\begin{array}{c}
B(p) \\
0
\end{array}\right] u_{c} \\
& \triangleq A_{c}(p) x_{c}+B_{c}(p) u_{c},
\end{aligned}
$$

where $A_{c}$ and $B_{c}$ are defined in (25). 
For the system (25), a stabilising control law exists if (Huang 2004).

$$
\operatorname{rank}\left[\begin{array}{ll}
A(p)-\lambda I & B(p) \\
H_{1} & 0
\end{array}\right]=4
$$

for all $\lambda$, which are the roots of the minimal polynomial of $A_{k f}$ (that is, for $\lambda=0$ and $\lambda= \pm j \omega_{f} l, l=1,2,3, \ldots, k$ ). Here $I$ denotes an identity matrix. Of course, the characteristic roots of $A_{k f}$ are the eigenvalues of the internal model matrix $G_{1}$. The matrices $A$ and $B$ of the augmented system (25) depend on the unknown parameter vector $p$. As such a feedback control law is obtained by the stabilisation of (25) at the chosen nominal (known) parameter value $p^{*}$. A stabilising feedback law takes the form

$$
u_{c}=-K_{1} x-K_{2} x_{s} \triangleq-K x_{c}
$$

where the gain vector $K$ can be computed using either pole assignment technique or the linear optimal control theory such that the closed-loop matrix $A_{c l}=\left[A_{c}\left(p^{*}\right)-\right.$ $\left.B_{c}\left(p^{*}\right) K\right]$ is Hurwitz.

Here, we design the control law using the optimal control theory. For optimal control, a quadratic performance index

$$
J=\int_{0}^{\infty}\left(x_{c}^{T} Q x_{c}+r u_{c}^{2}\right) d t
$$

is chosen, where the weighting matrix $Q$ is a positive definite symmetric matrix and $r>0$. The optimal gain vector is given by

$$
K=r^{-1} B_{c}^{T}\left(p^{*}\right) P,
$$

where $P$ is the positive definite symmetric matrix, which satisfies the algebraic Riccati equation (Kailath 1980)

$$
\begin{aligned}
& A_{c}^{T}\left(p^{*}\right) P+P A_{c}\left(p^{*}\right) \\
& \quad-P B_{c}\left(p^{*}\right) r^{-1} B_{c}^{T}\left(p^{*}\right) P+Q=0 .
\end{aligned}
$$

The weighting matrix $Q$ and $r$ can be chosen to shape the transient responses.

Although the gain vector $K$ is computed for a known nominal value of $w$, it follows that the closed-loop matrix $A_{c l}(p)$ remains Hurwitz for perturbations $\tilde{p} \in \tilde{\Omega}_{p}$, where $\tilde{\Omega}_{p}$ is a sufficiently small open set. Thus it follows that the origin $x_{c}=0$ of the non-linear system (24) is exponentially stable for $v=0$ and for sufficiently small $\tilde{p}$. In the closed-loop system including the internal model of the $k$-fold exosystem, one can show that $Y_{l p}$ in the Taylor series expansion of the tracking error (16) are null vectors for small $\tilde{p}$ and $l=1, \ldots, k$. Thus the tracking error $e(t)$ satis- fies (13) as $t \rightarrow \infty$, and therefore, the steady-state tracking error is zero up to $k$ th order.

We may point out that although this paper treats pectoral fin control of a BAUV, the design methodology developed here is applicable to other swimming and flying robotic systems, which employ oscillating control surfaces (flapping fins and wings) to produce propulsive and manoeuvering forces. The control system designed here can be synthesised using the state variables of the robotic vehicle, and the linear servo compensator forms a part of the controller. Although, the stabiliser is designed based on the linearised model, the linear quadratic design does provide robustness to parametric uncertainties by the choice of suitable performance index. This is of practical importance because system parameters are not precisely known.

\section{Simulation results}

In this section, simulation results for the closed-loop system (4), (21) and (27) using MATLAB/SIMULINK are presented. The parameters of the vehicle model in Equation (4) are taken from Singh et al. (2004). The BAUV is assumed to be moving with a constant forward velocity of $0.8 \mathrm{~m} / \mathrm{s}$. The vehicle parameters are $l=1.282 \mathrm{~m}, m=4.1548 \mathrm{~kg}$, $I_{y}=0.5732 \mathrm{~kg} \cdot \mathrm{m}^{2}, x_{G}=0$ and $Z_{G}=0.578802 \times 10^{-8}$ $\mathrm{m}$. The hydrodynamic parameters for the forward velocity of $0.8 \mathrm{~m} / \mathrm{s}$ are $z_{\dot{q}}^{\prime}=-0.825 \times 10^{-5}, z_{\dot{w}}^{\prime}=-0.825 \times$ $10^{-5}, z_{q}^{\prime}=-0.238 \times 10^{-2}, z_{w}^{\prime}=-0.738 \times 10^{-2}, M_{\dot{q}}^{\prime}=$ $-0.16 \times 10^{-3}, \quad M_{\dot{w}}^{\prime}=-0.825 \times 10^{-5}, M_{q}^{\prime}=-0.117 \times$ $10^{-2}$ and $M_{w}^{\prime}=0.314 \times 10^{-2}$. The pectoral fins are attached at a distance of $d_{c g f}=0.15 \mathrm{~m}$. The simulation results are obtained for fin oscillation frequencies of $f=6$ $\mathrm{Hz}$ and $f=8 \mathrm{~Hz}$. Of course, the controller design is applicable for any choice of frequency of oscillation of the fins.

Using CFD analysis, the fin forces and the moments coefficients have been obtained for a fixed Strouhal number $S_{t}=0.6$, where $S_{t}=\frac{c f}{U_{\infty}}, U_{\infty}$ is $0.8 \mathrm{~m} / \mathrm{s}, f$ is the frequency of the fin oscillation and $c$ is the chord of the foil. The parameter vectors $f_{a}, f_{b}, m_{a}$ and $m_{b}$ used for simulations are

$$
\begin{aligned}
f_{a} & =\left(f_{0}^{c}(0), f_{1}^{s}(0), f_{1}^{c}(0), \ldots, f_{M}^{s}(0), f_{M}^{c}(0)\right)^{T}, \\
f_{b} & =\left(\frac{\partial f_{0}^{c}}{\partial \beta}(0), \frac{\partial f_{1}^{s}}{\partial \beta}(0), \frac{\partial f_{1}^{c}}{\partial \beta}(0), \ldots, \frac{\partial f_{s}^{M}}{\partial \beta}(0), \frac{\partial f_{c}^{M}}{\partial \beta}(0)\right)^{T}, \\
m_{a} & =\left(m_{0}^{c}(0), m_{1}^{s}(0), m_{1}^{c}(0), \ldots, m_{M}^{s}(0), m_{M}^{c}(0)\right)^{T}, \\
m_{b} & =\left(\frac{\partial m_{0}^{c}}{\partial \beta}(0), \frac{\partial m_{1}^{s}}{\partial \beta}(0), \frac{\partial m_{1}^{c}}{\partial \beta}(0), \ldots, \frac{\partial m_{s}^{M}}{\partial \beta}(0), \frac{\partial m_{c}^{M}}{\partial \beta}(0)\right)^{T},
\end{aligned}
$$

where $f_{a}, f_{b}, m_{a}, m_{b} \in R^{2 M+1}$. In the Fourier expansion, four harmonic components, which are dominant, are 

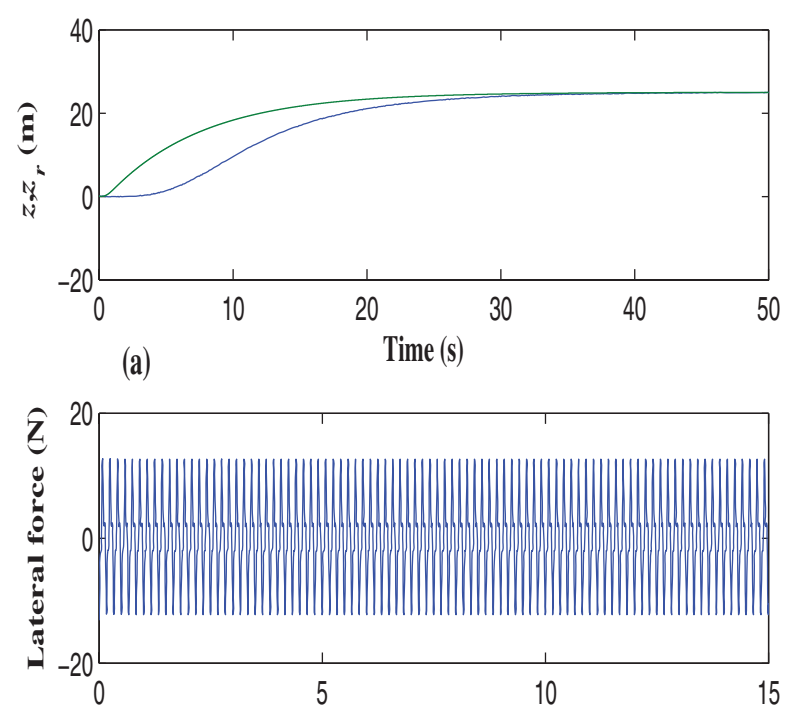

(c)

Time(s)

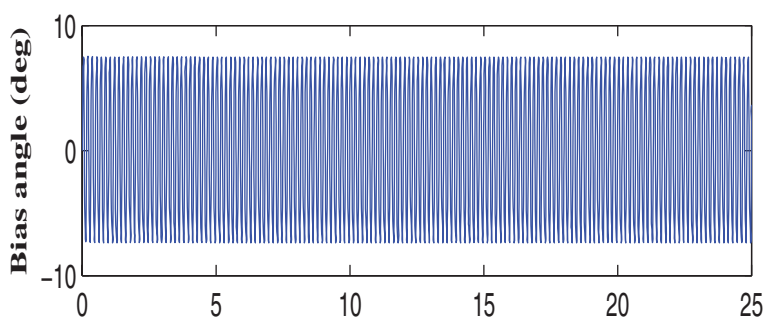

(e)

Time(s)

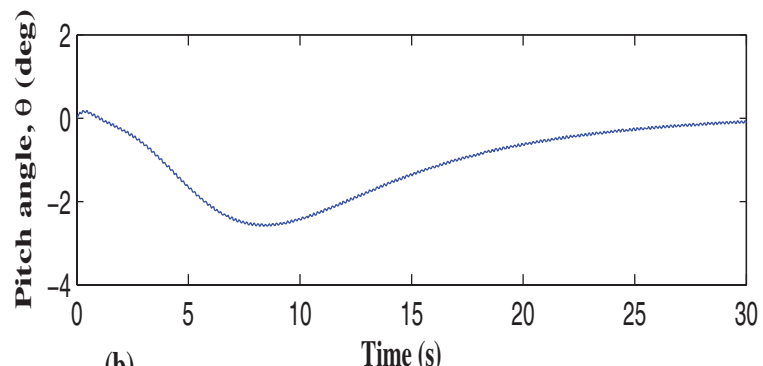

(b)

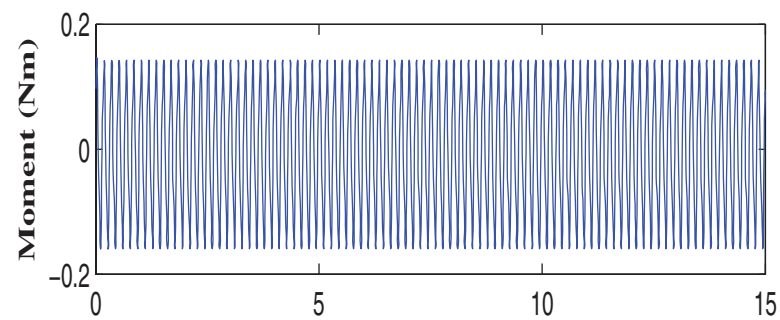

(d)

Time (s)

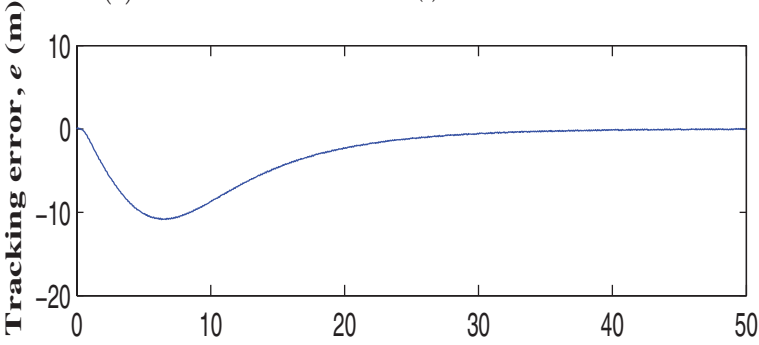

(f)

Time (s)

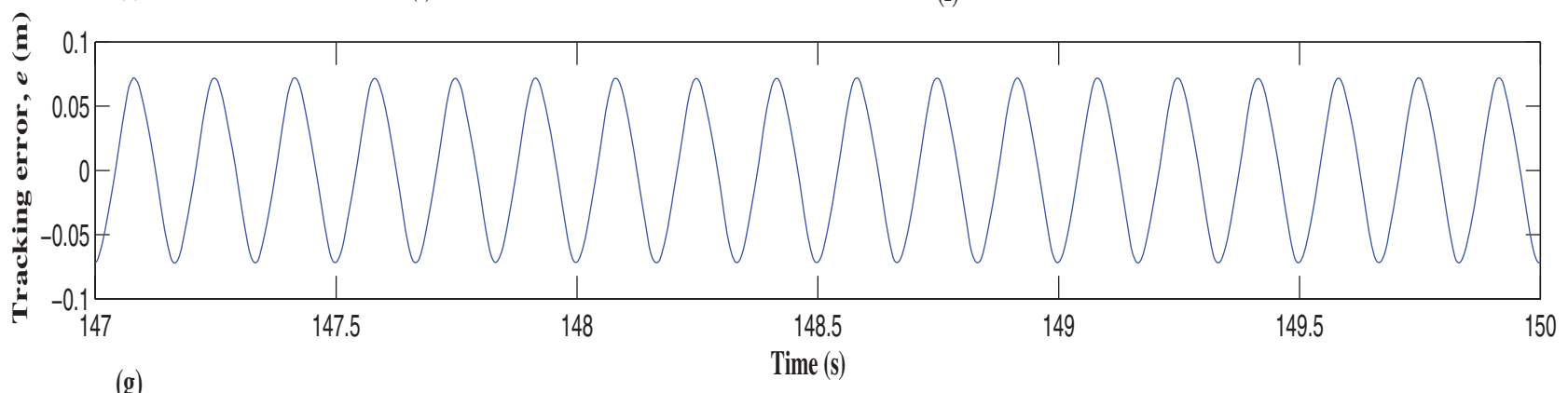

(g)

Figure 2. BAUV control using first-order servo compensator $-\omega_{f}=6 \mathrm{~Hz}$ and the nominal parameters: (a) dive plane depth, $z$, and reference depth, $z_{r}$ (in metres), (b) pitch angle, $\theta$ (in degrees), (c) lateral force (in Newtons), (d) moment (in Newton metres), (e) bias angle (in degrees), (f) tracking error, $e$ (in metres), (g) tracking error plotted for smaller time interval, $e$ (in metres).

retained; that is, $M=4$. Then the values of $f_{a}, f_{b}, m_{a}, m_{b}$ for $\mathrm{M}=4$ are

$$
\begin{aligned}
f_{a}= & (0,-40.0893,-43.6632,-0.3885,0.6215,6.2154, \\
& -10.1777,-0.1554,0.6992), \\
f_{b}= & (68.9975,0.4451,-16.4704,64.1009,-19.5864, \\
& -0.8903,-2.2257,2.2257,4.8966),
\end{aligned}
$$

$$
\begin{aligned}
m_{a}= & (0,0.6037,0.4895,0,-0.0054,0, \\
& -0.0925,0,-0.0054), \\
m_{b}= & (-0.4986,-0.3739,-0.0935,-0.2493,0.1246, \\
& 0.0312,-0.0312,0.0935,0) .
\end{aligned}
$$

(Readers may refer to Narasimhan et al. [2006] for the details.) It is pointed out that these parameters are obtained using the Fourier decomposition of the fin force and moment, 

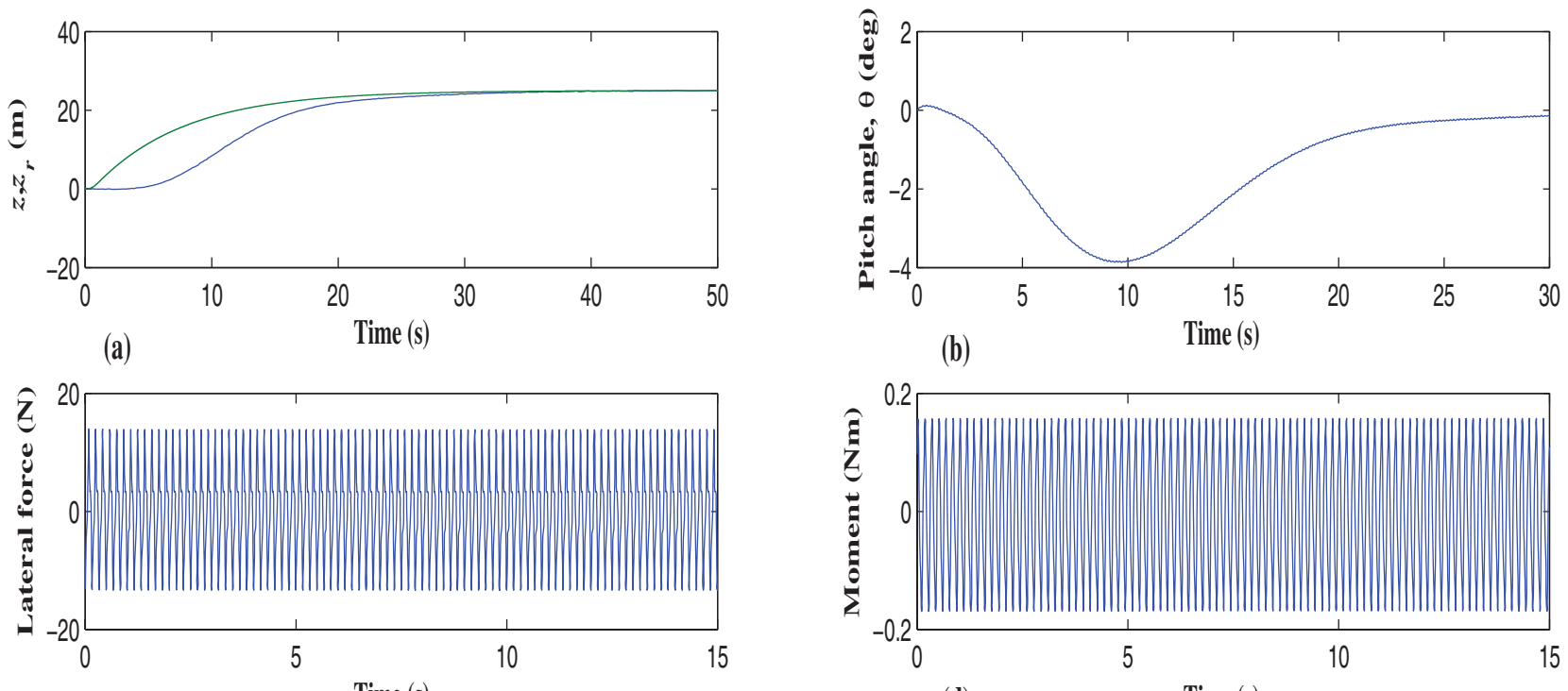

(c)

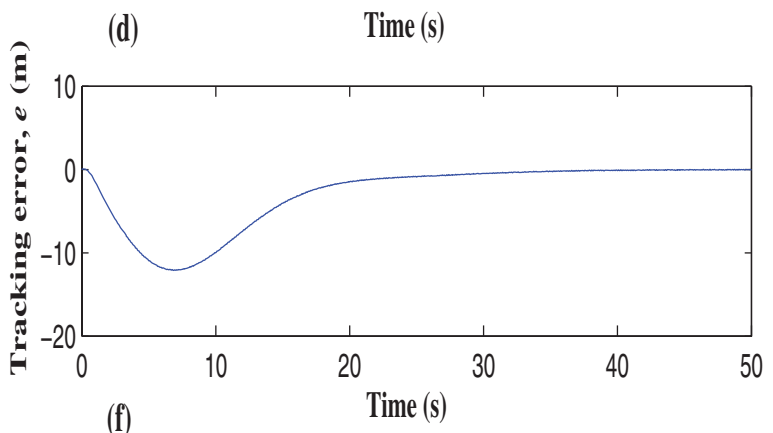

(e)

Time (s)
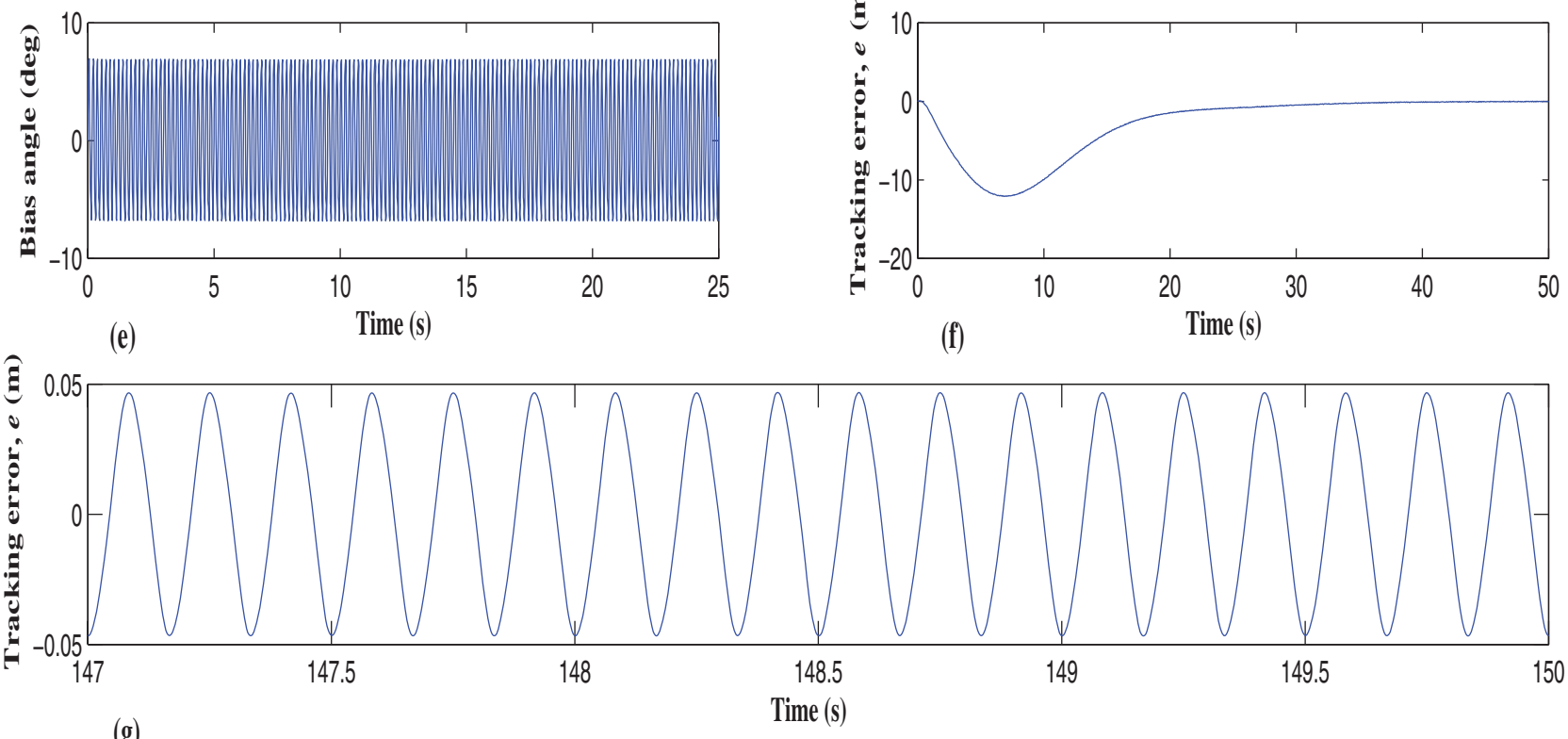

(g)

Figure 3. BAUV control using first-order servo compensator $-\omega_{f}=6 \mathrm{~Hz},-25 \%$ uncertainty: (a) dive plane depth, $z$, and reference depth, $z_{r}$ (in metres), (b) pitch angle, $\theta$ (in degrees), (c) lateral force (in Newtons), (d) moment (in Newton metres), (e) bias angle (in degrees), (f) tracking error, $e$ (in metres), (g) tracking error plotted for smaller time interval, $e$ (in metres).

and are computed by multiplying the Fourier coefficients by $\frac{1}{2} \rho W_{a} U_{\infty}^{2}$ and $\frac{1}{2} \rho W_{a} c U_{\infty}^{2}$, respectively, where $W_{a}$ is the surface area of the foil. For simulation, the initial conditions of the vehicle are assumed to be $x(0)=0$ and $x_{s}(0)=0$.

A smooth reference trajectory $z_{r}(t)$ converging to $z^{*}$, the target depth, using a fourth-order filter

$$
G_{c}(s)=\frac{\lambda_{1} \omega_{n c}^{2}}{\left(s+\lambda_{c 1}\right)\left(s+\lambda_{c 2}\right)\left(s^{2}+2 \zeta_{c} \omega_{n c} s+\omega_{n c}^{2}\right)}
$$

is generated, where $\omega_{n c}=4.95, \zeta_{c}=0.707, \lambda_{c 1}=0.14$ and $\lambda_{c 2}=3.5$ are the real poles.
A simple servo compensator of first order providing integral error feedback, as well as a compensator representing the internal model of $t w o$-fold exosystem are designed. The latter servo compensator is of fifth order. For the first-order compensator, $G_{1}=0$ and $G_{2}=0.5$, and for the fifth order, one has

$$
\begin{aligned}
d G_{1} & =\operatorname{diag}\left(0,\left[\begin{array}{ll}
0 & -\omega_{f} \\
\omega_{f} & 0
\end{array}\right],\left[\begin{array}{ll}
0 & -2 \omega_{f} \\
2 \omega_{f} & 0
\end{array}\right]\right), \\
G_{2} & =[0.5,0,0.5,0,0.5]^{T} .
\end{aligned}
$$



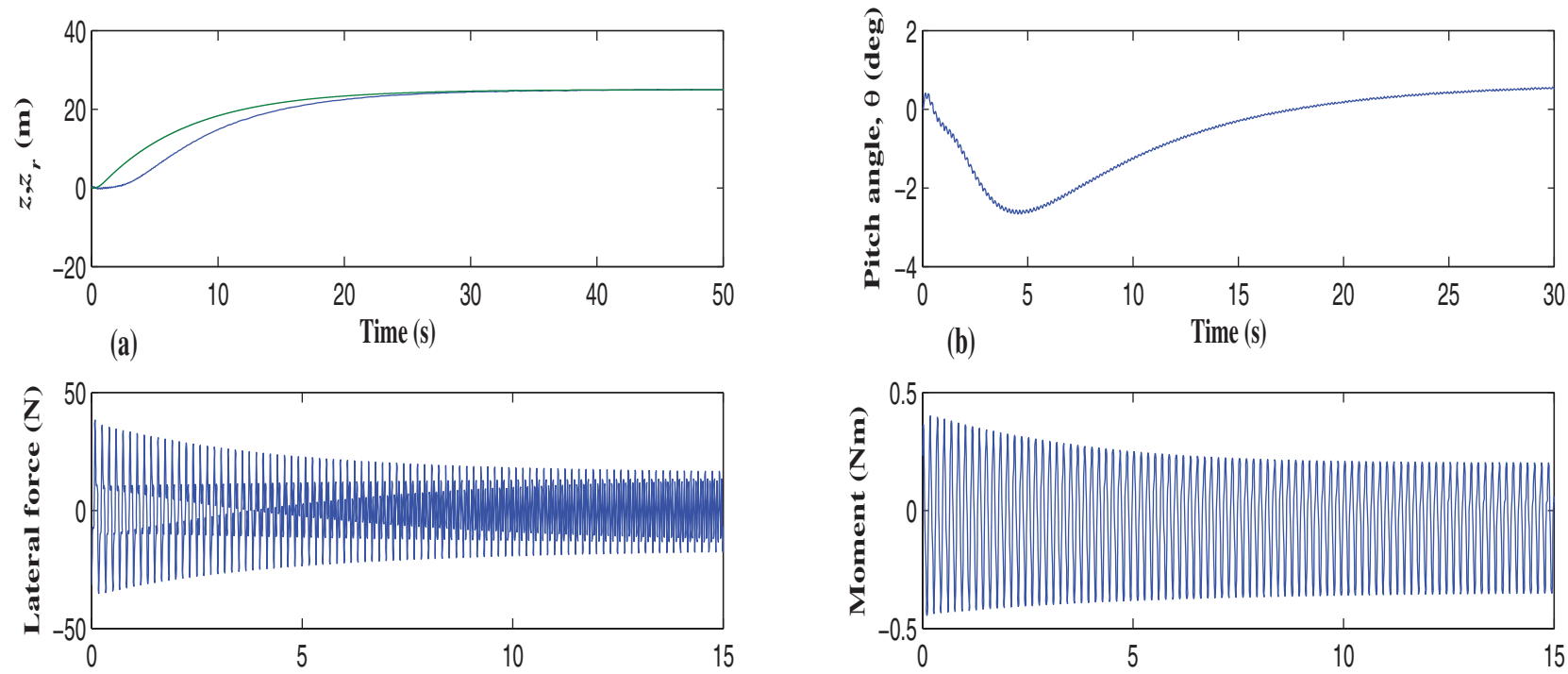

(c)

Time (s)

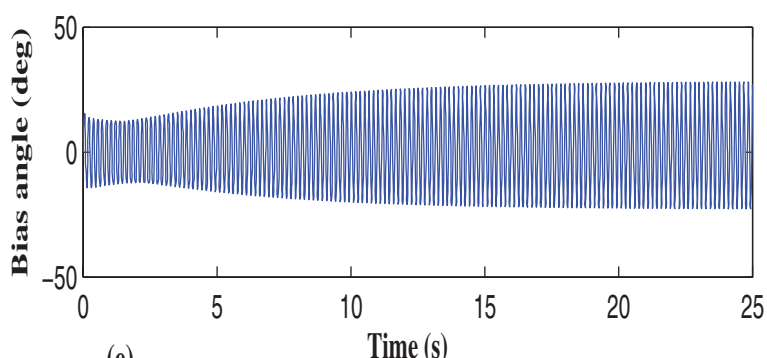

(e)

Time (s)

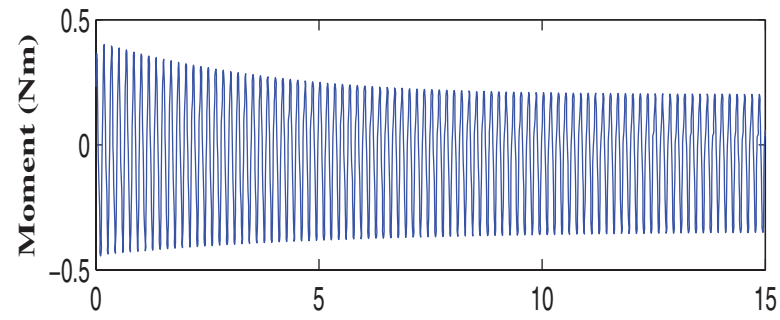

(d)

Time (s)
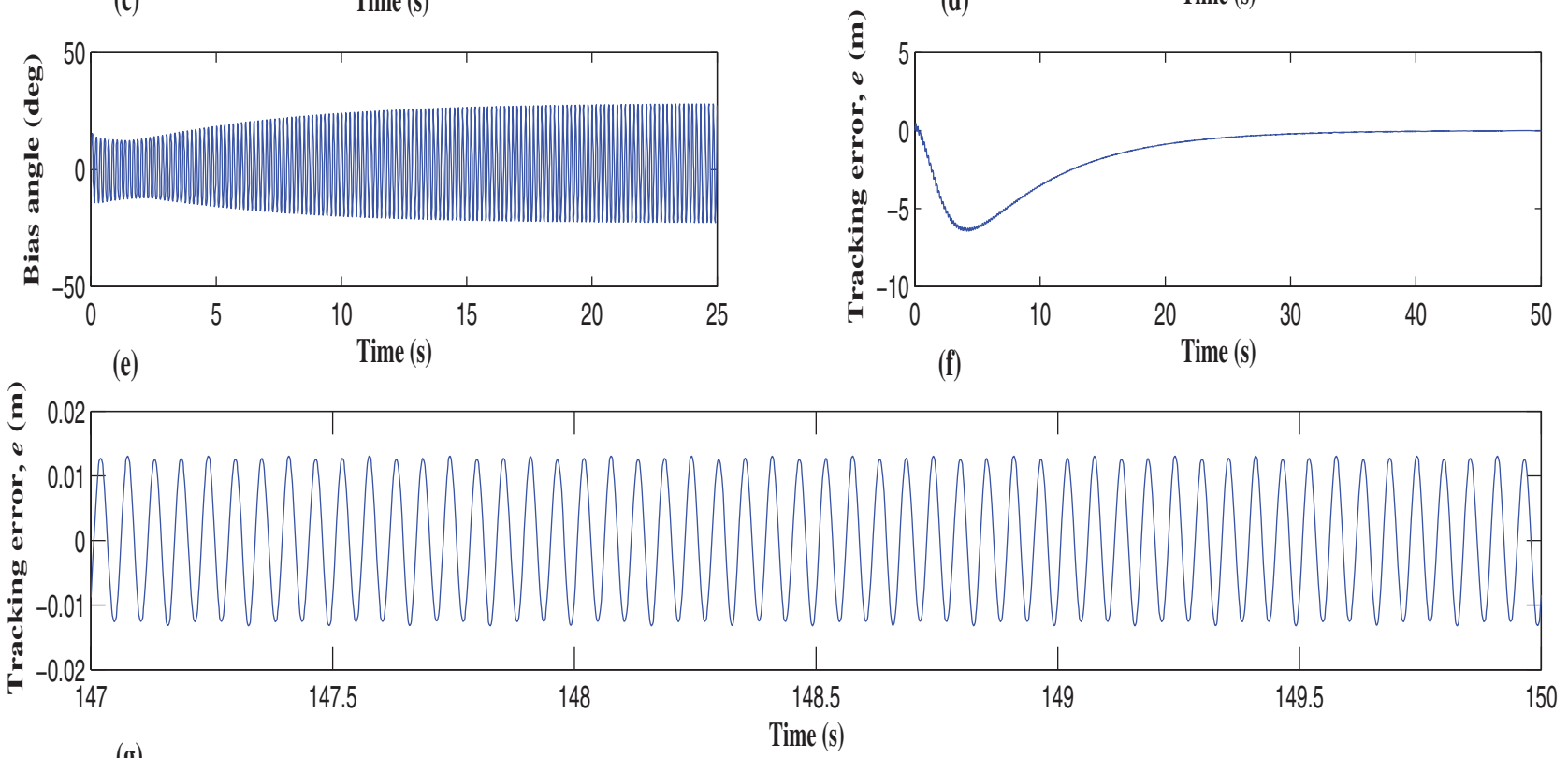

(g)

Figure 4. BAUV control using internal model of two-fold exosystem $-\omega_{f}=6 \mathrm{~Hz}$ and the nominal parameters: (a) dive plane depth, $z$, and reference depth, $z_{r}$ (in metres), (b) pitch angle, $\theta$ (in degrees), (c) lateral force (in Newtons), (d) moment (in Newton meter), (e) bias angle (in degrees), (f) tracking error, $e$ (in metres), (g) tracking error plotted for smaller time interval, $e$ (in metres).

If the input $e$ to the servo compensator is zero, then the first-order system generates constant trajectory, but the fifth-order servo compensator can generate trajectories of the form $c_{1}+c_{2} \sin \left(\omega_{f} t+\theta_{1}\right)+c_{3} \sin \left(2 \omega_{f} t+\theta_{2}\right)$ by appropriate choice of initial conditions. For the control law design, linear optimal control theory is used. For the first-order compensator, $Q=1$, and for the fifth order, $Q$ is an identity matrix of dimension $9 \times 9$, and $r=0.0001$.

\section{Case I: BAUV control using first-order servo compensator: $\omega_{f}=6 \mathrm{~Hz}$, nominal parameters}

The complete closed-loop system including the nominal BAUV model and the first-order servo compensator is simulated for a fin oscillation frequency of $6 \mathrm{~Hz}$. A smooth reference trajectory $z_{r}(t)$ converging to $z^{*}=25 \mathrm{~m}$ is generated. Thus it is desired to steer the BAUV to a depth of $25 \mathrm{~m}$. The optimal controller gains are computed for the nominal BAUV model. Selected responses are shown in 

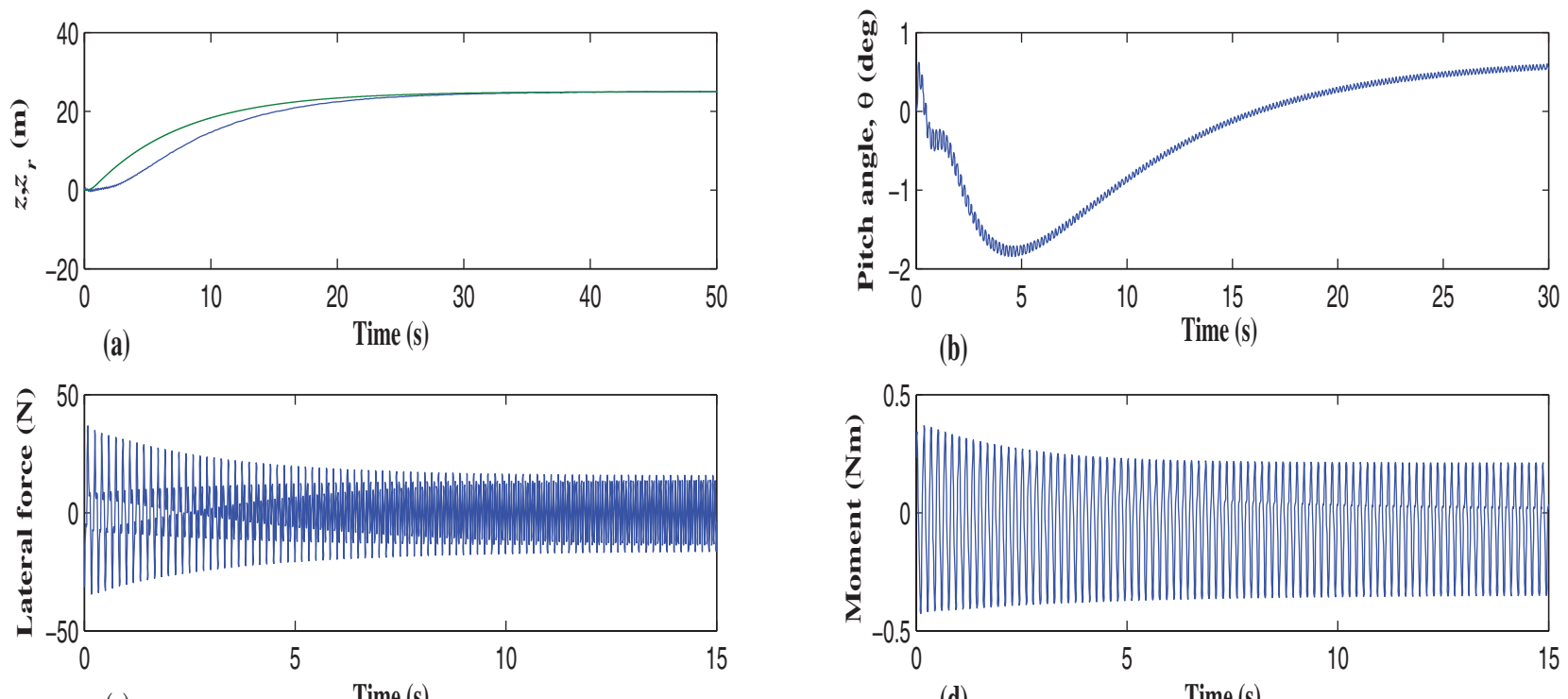

(c)

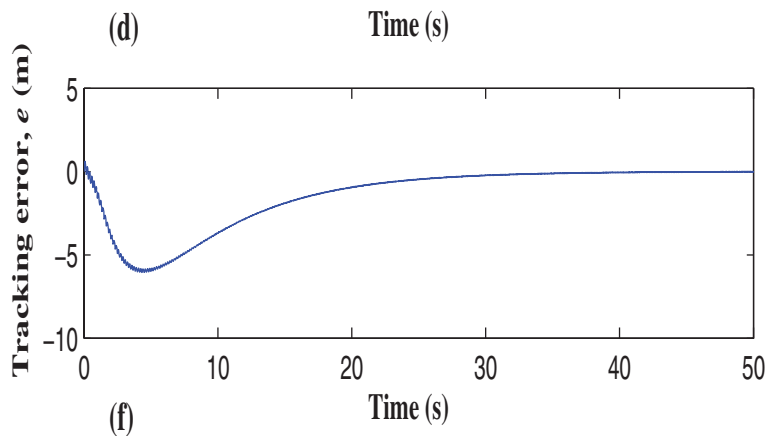

(e)

Time (s)

(f)

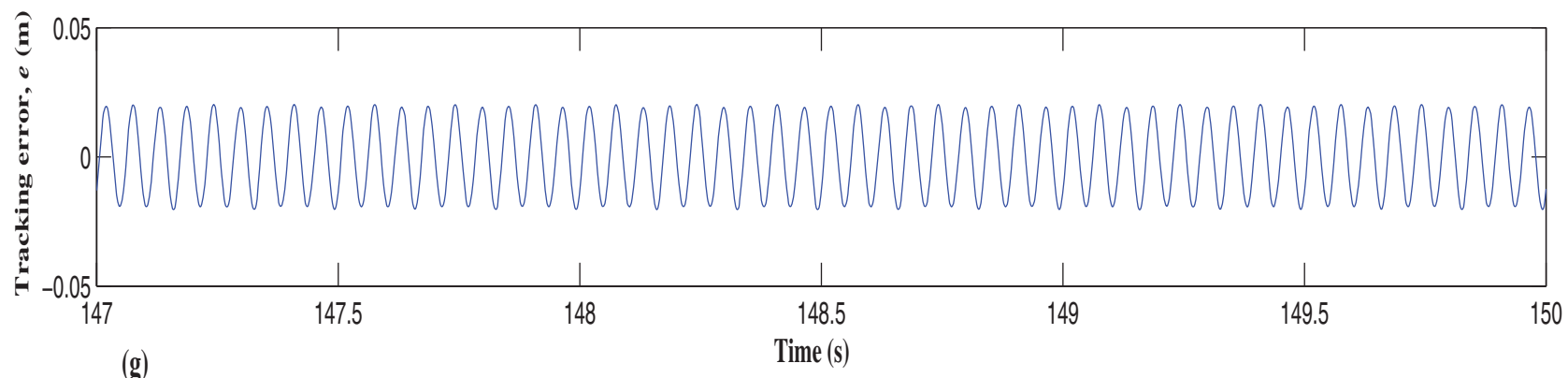

Figure 5. BAUV control using internal model of two-fold exosystem $-\omega_{f}=6 \mathrm{~Hz},+25 \%$ uncertainty: (a) dive plane depth, $z$, and reference depth, $z_{r}$ (in metres), (b) pitch angle, $\theta$ (in degrees), (c) lateral force (in Newtons), (d) moment (in Newton metres) (e) bias angle (in degrees) (f) tracking error, $e$ (in metres) (g) tracking error plotted for smaller time interval, $e$ (in metres).

Figure 2. It is observed that the BAUV attains the target depth in little over $30 \mathrm{~s}$. In steady-state, fin forces, moment and bias angle exhibit bounded periodic oscillations. Note that the chosen servo compensator has a simple pole at zero and as such it can only suppress any non-zero bias in the tracking error. We observe that indeed average tracking error is zero, but periodic oscillations including the fundamental component $(6 \mathrm{~Hz})$ and higher harmonics persist. The magnitude of the tracking error $e$ is observed to be around $0.08 \mathrm{~m}$. The maximum control magnitude is around $8^{\circ}$. The peak control force needed is $12 \mathrm{~N}$ and the control moment is $0.15 \mathrm{~N} \cdot \mathrm{m}$.

\section{Case II: BAUV control using first-order servo compensator: $\omega_{f}=6 \mathrm{~Hz},-25 \%$ uncertainty}

Now simulation is done to examine the effect of uncertainties in the control force and moment coefficients. For this purpose the elements of the vectors $f_{a}, f_{b}, m_{a}$ and $m_{b}$ are perturbed by a factor of 0.75 for simulation; that is, the perturbed values of these vectors are $25 \%$ lesser than the nominal values. However, the controller gains used in Figure 2 computed for the nominal values are retained. Selected plots are provided in Figure 3. It is again noted that with the servo compensator of first order, the oscillatory components of the tracking error are not suppressed. But, 

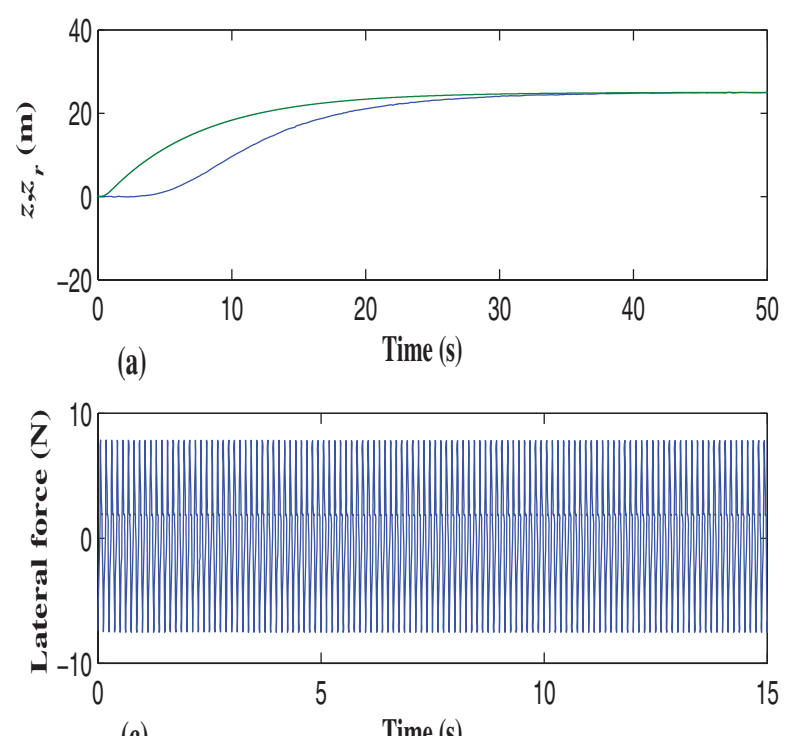

(c)

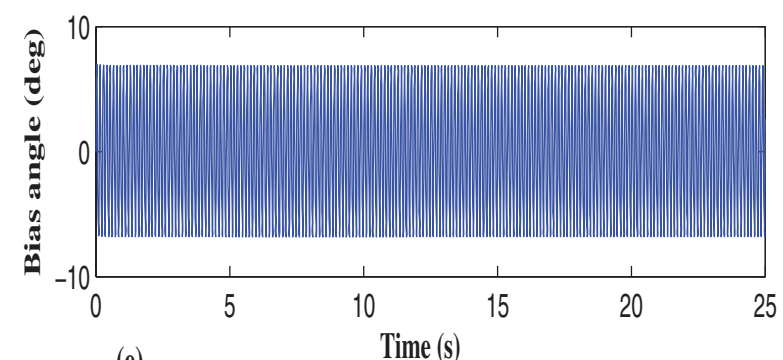

(e)
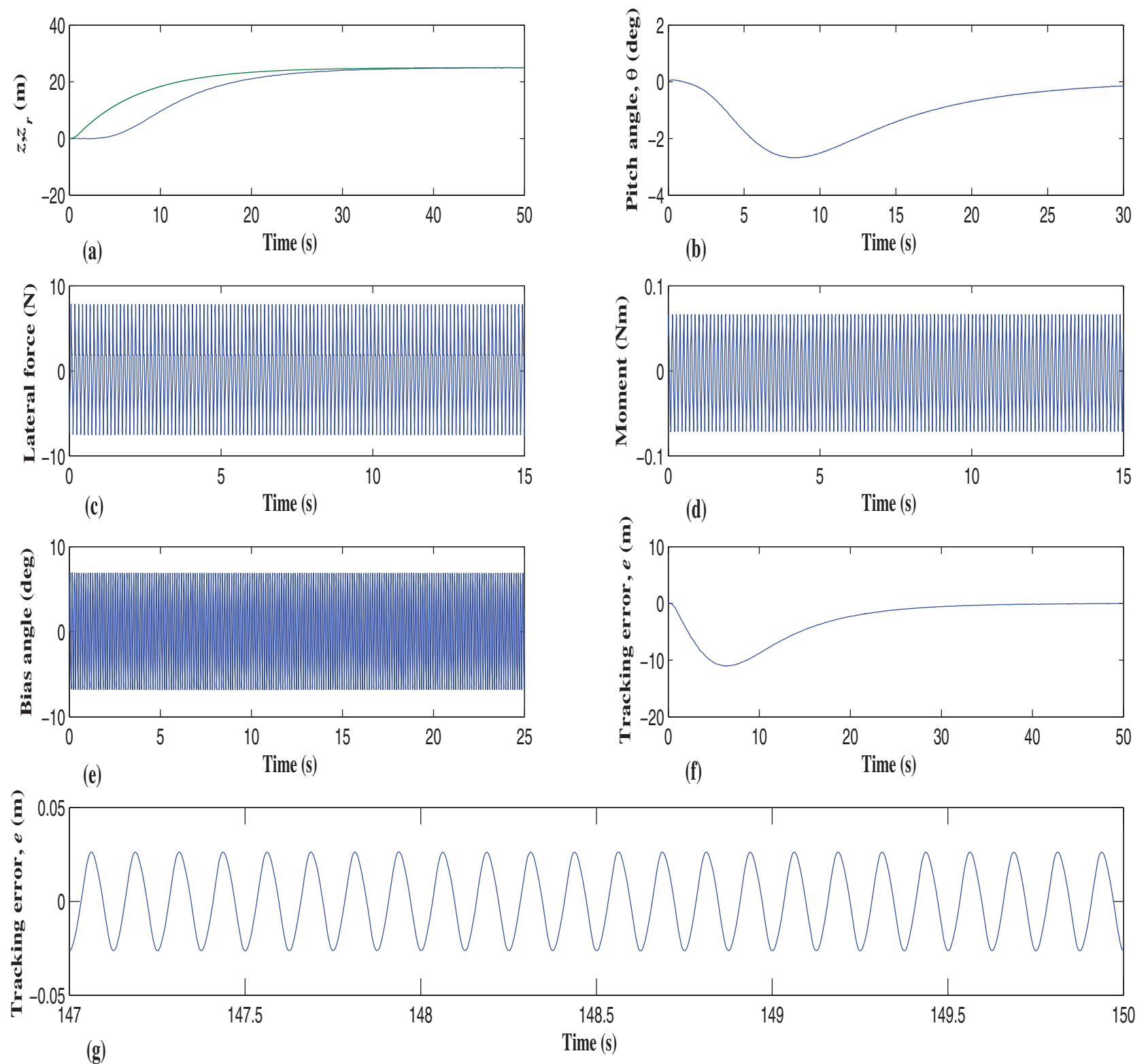
-

Figure 6. BAUV control using first-order servo compensator $-\omega_{f}=8 \mathrm{~Hz}$ and the nominal parameters: (a) dive plane depth, $z$, and reference depth, $z_{r}$ (in metres), (b) pitch angle, $\theta$ (in degrees), (c) lateral force (in Newtons), (d) moment (in Newton metres), (e) bias angle (in degrees), (f) tracking error, $e$ (in metres), (g) tracking error plotted for smaller time interval, $e$ (in metres).

the average value of the tracking error is zero. The magnitude of the tracking error is $0.05 \mathrm{~m}$. The maximum control magnitude and the response time to attain the target depth are of the same order as in Figure 2.

Simulation is also done for fin force coefficients with $+25 \%$ uncertainty (perturbed values are 1.25 times the nominal values) using the nominal controller. In this case also, a smooth control to the desired depth is accomplished. (In order to save space, the results are not shown here).

\section{Case III: BAUV control using internal model of} 2-fold exosystem: $\omega_{f}=6 \mathrm{~Hz}$, nominal parameters

For attenuating the dominant oscillatory components of the tracking error, it is essential to synthesise servo compensator of higher order. For the purpose of illustration, a servo compensator of fifth order is deigned using internal model of two-fold exosystem. Selected responses for the nominal BAUV model are shown in Figure 4. It is seen that desired depth is smoothly attained. The magnitude of the tracking error is significantly smaller compared with that of Figure 2 . 

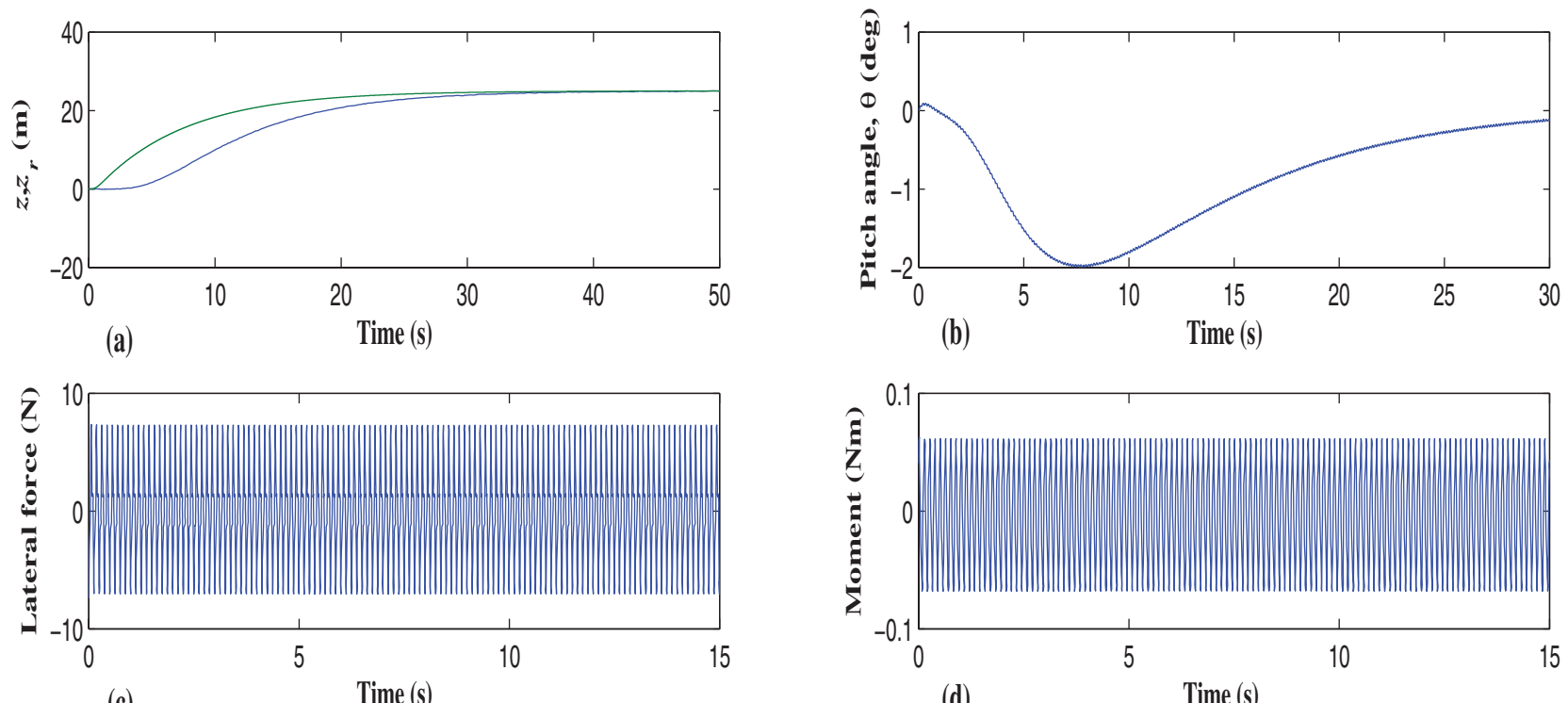

(c)
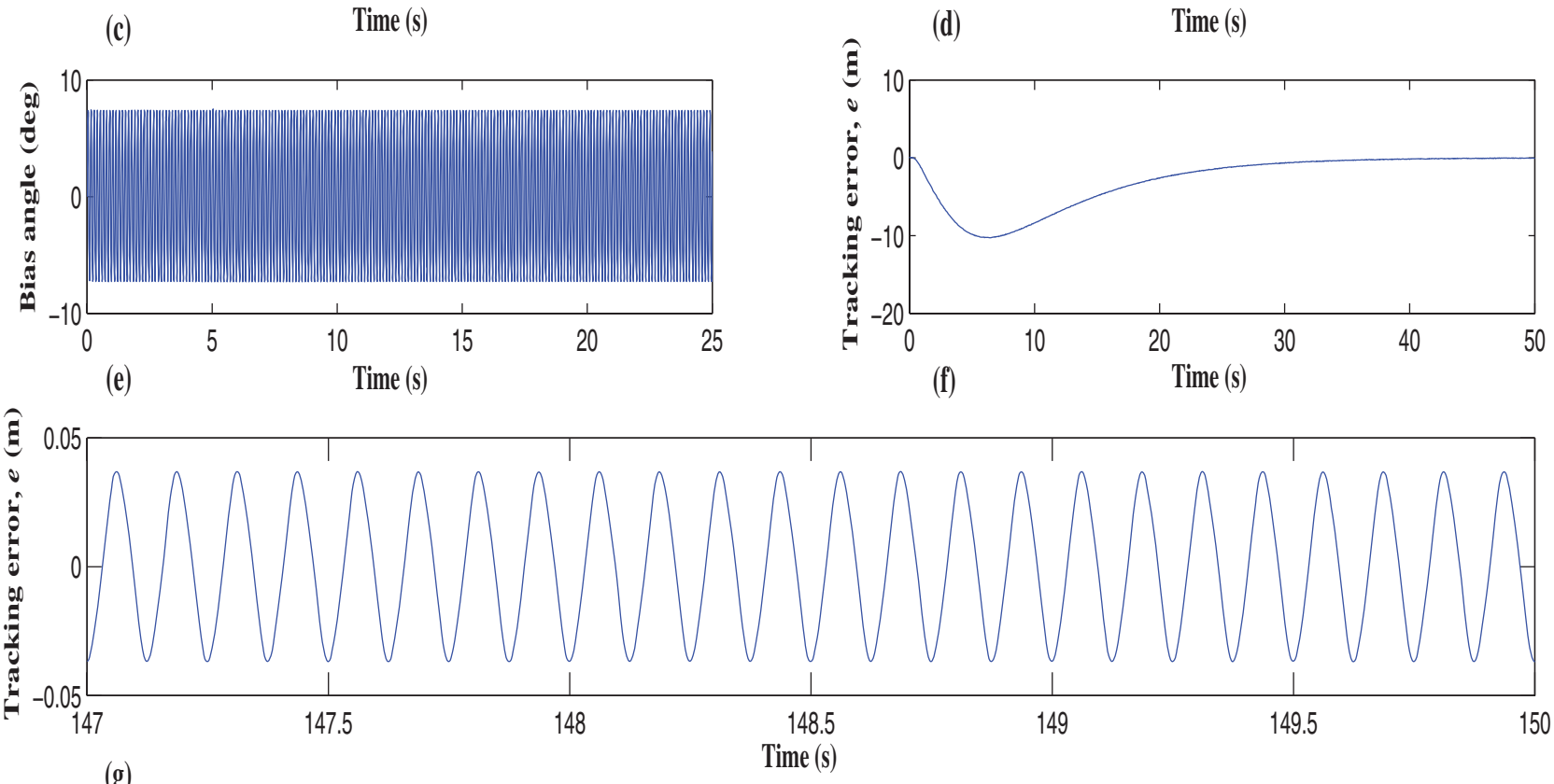

Figure 7. BAUV control using first-order servo compensator $\omega_{f}=8 \mathrm{~Hz},+25 \%$ uncertainty: (a) dive plane depth, $z$, and reference depth, $z_{r}$ (in metres), (b) pitch angle, $\theta$ (in degrees), (c) lateral force (in Newtons), (d) moment (in Newton metres), (e) bias angle (in degrees), (f) tracking error, $e$ (in metres), (g) tracking error plotted for smaller time interval, $e$ (in metres).

Interestingly, the designed servo compensator suppresses the constant bias, fundamental and second harmonic components in the tracking error response, and only oscillations of frequency $18 \mathrm{~Hz}$ and higher remain. Although one can design a higher-order compensator, we observe that even this fifth-order servo compensator yields maximum error little over $0.01 \mathrm{~m}$, which is negligible for practical purposes. The maximum control magnitude is observed to be around $30^{\circ}$. The target depth is attained in a little over $30 \mathrm{~s}$ as in Figure 2.

\section{Case IV: BAUV control using internal model of} two-fold exosystem: $\omega_{f}=6 \mathrm{~Hz},+25 \%$ uncertainty

Now simulation is done to examine the robustness of the control system. It is assumed that the fin force coefficients are $25 \%$ greater than the nominal values, but the nominal control system used for Figure 4 is retained. Selected responses are shown in Figure 5. Similar to Figure 4, we observe that the vehicle attains the desired depth and controller is able to suppress the bias, fundamental and second harmonics in the tracking error response. Compared with 

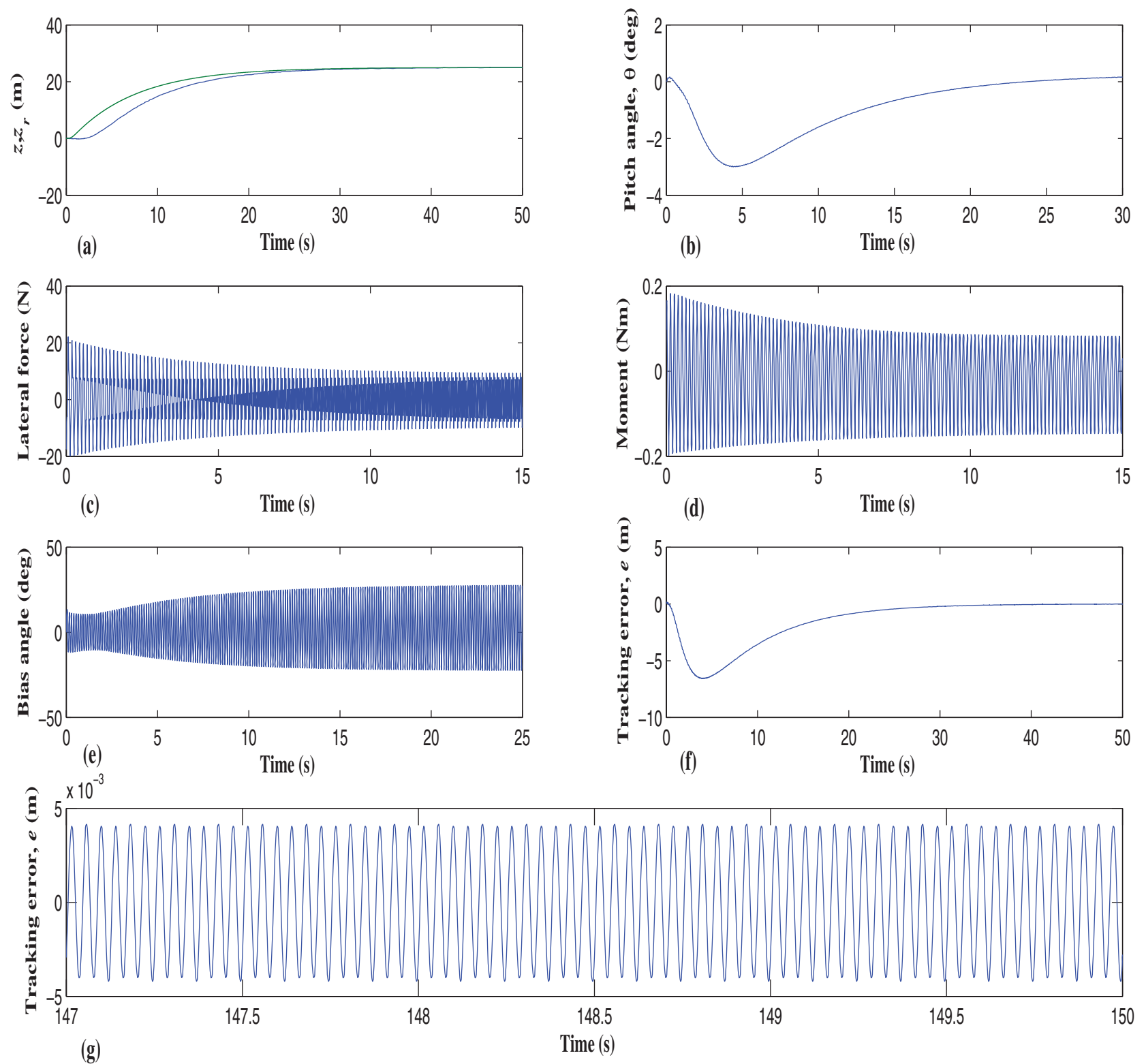

Figure 8. BAUV control using internal model of two-fold exosystem $-\omega_{f}=8 \mathrm{~Hz}$ and the nominal parameters: (a) dive plane depth, $z$, and reference depth, $z_{r}$ (in metres), (b) pitch angle, $\theta$ (in degrees), (c) lateral force (in Newtons), (d) moment (in Newton metres), (e) bias angle (in degrees), (f) tracking error, e (in metres), (g) tracking error plotted for smaller time interval, $e$ (in metres).

Figure 4, the tracking error magnitude in Figure 5 is a little higher at $0.02 \mathrm{~m}$, but the maximum control magnitude and the target depth response time are of the same magnitude.

\section{Case V: BAUV control using first-order servo compensator: $\omega_{f}=8 \mathrm{~Hz}$, nominal parameters}

The frequency of the pectoral fin oscillation is set at $8 \mathrm{~Hz}$ and the performance of the first-order servo compensator is evaluated. Of course, for $8 \mathrm{~Hz}$ the fin force and mo- ment coefficients have changed, and the feedback gains of the controller are redesigned using the same values of $Q$ and $r$. The responses are shown in Figure 6. It is observed that the average tracking error is zero, but the oscillatory components in the tracking error are still present. The simulation results shown in Figure 6 are some what similar to the results obtained in Figure 2. Though the tracking error pattern in Figures 2 and 6 are the same, the magnitude of the tracking error in Figure 6 is $0.025 \mathrm{~m}$, lesser than that in Figure 2. The maximum control magnitude and the target 

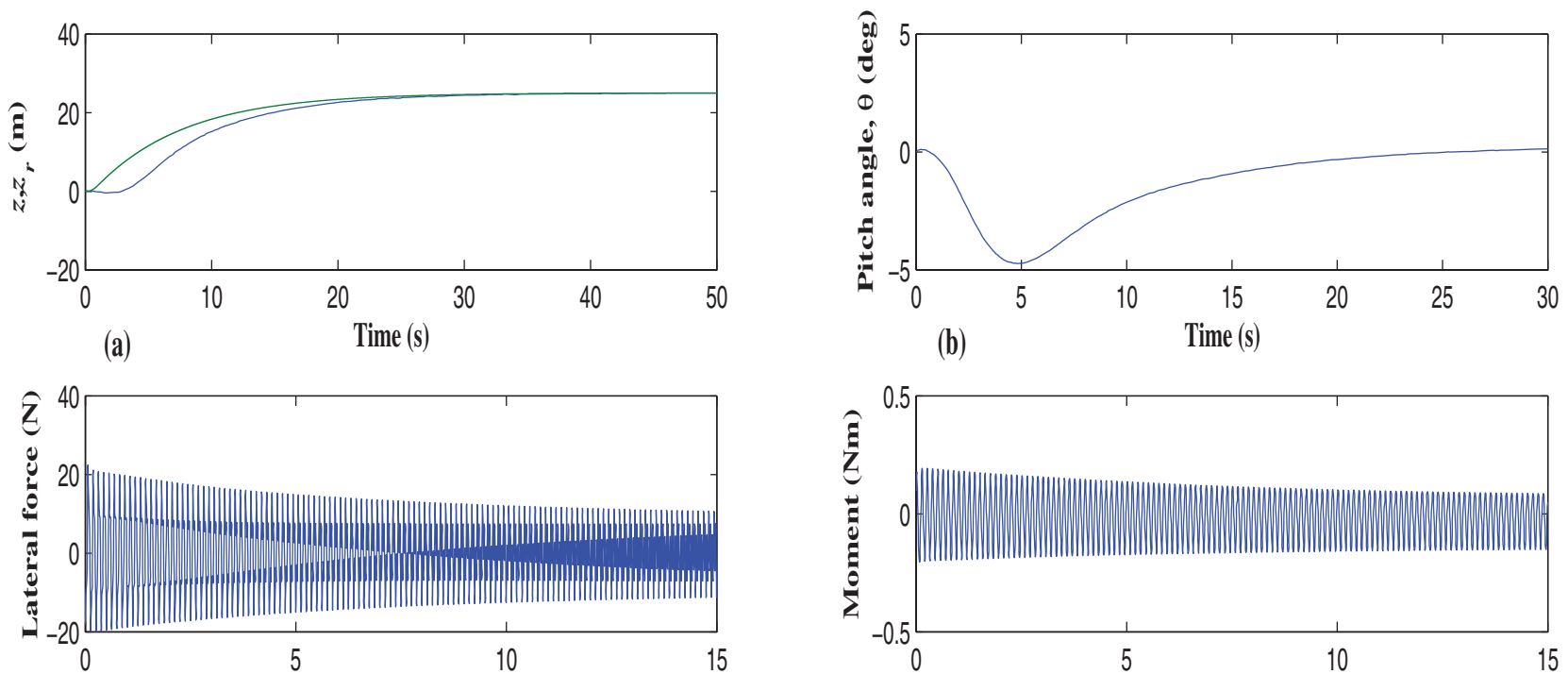

(c)

Time (s)

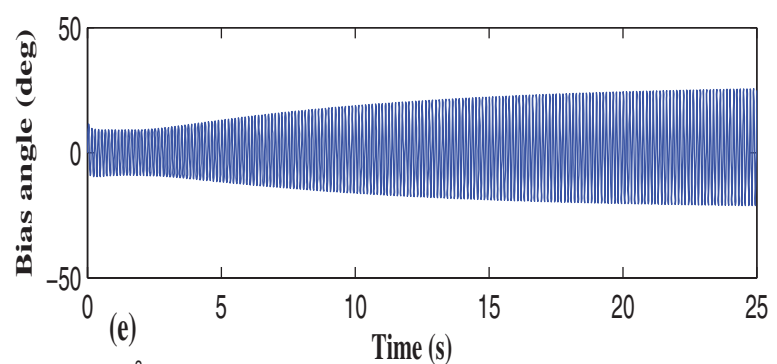

(d)

Time (s)
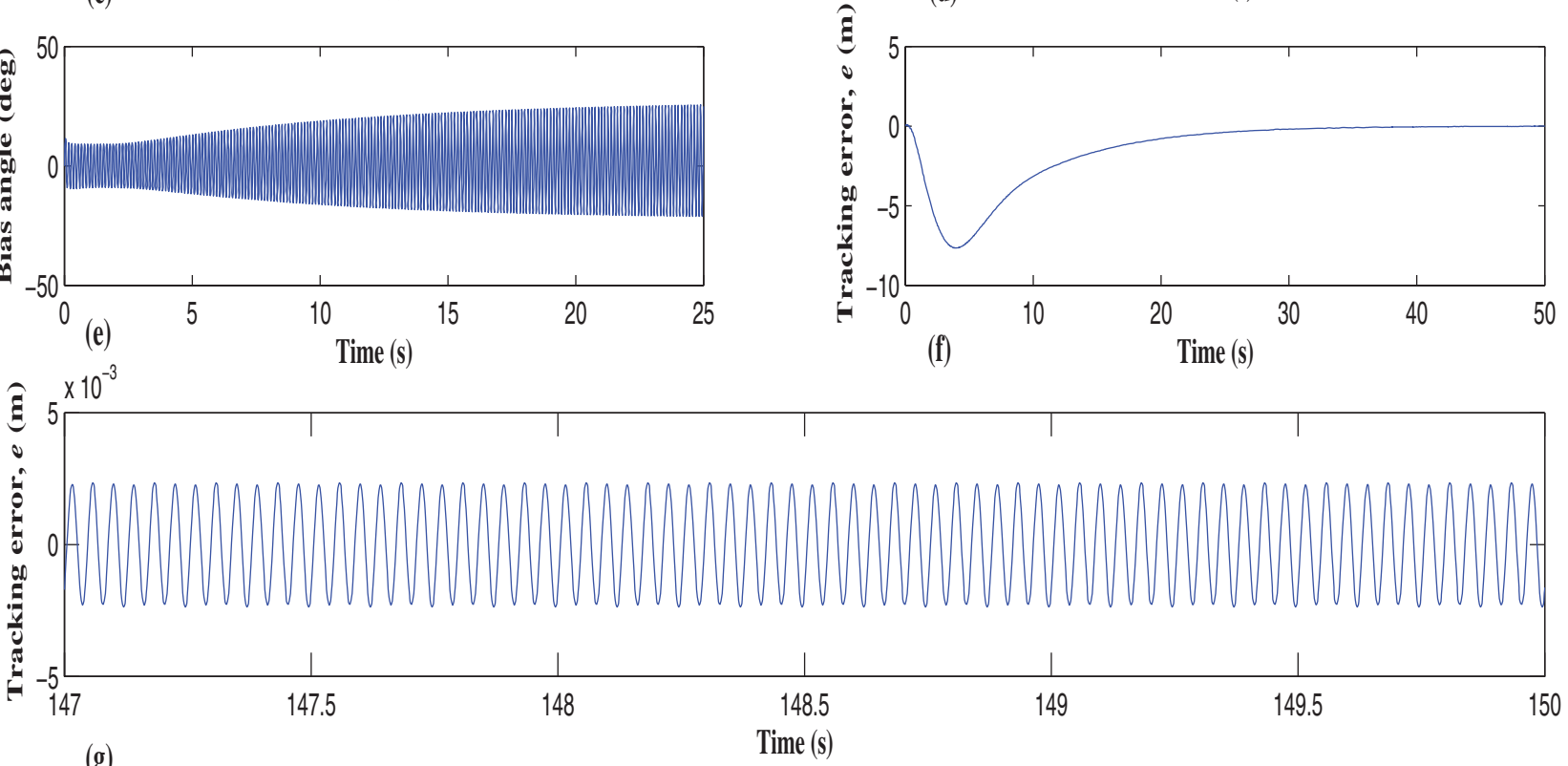

(g)

Figure 9. BAUV control using internal model of two-fold exosystem $-\omega_{f}=8 \mathrm{~Hz},-25 \%$ uncertainty: (a) dive plane depth, $z$, and reference depth, $z_{r}$ (in metres), (b) pitch angle, $\theta$ (in degrees), (c) lateral force (in Newtons), (d) moment (in Newton metres), (e) bias angle (in degrees), (f) tracking error, $e$ (in metres), (g) tracking error plotted for smaller time interval, $e$ (in metres).

depth response time in both Figures 2 and Figure 6 are almost the same.

\section{Case VI: BAUV control using first-order servo compensator: $\omega_{f}=8 \mathrm{~Hz},+25 \%$ uncertainty}

An uncertainty of $+25 \%$ is introduced in the fin force coefficents for simulation. The results using the nominal controller of Figure 6 are shown in Figure 7. The magnitude of the tracking error in Figure 7 is 0.04 m, whereas in Figure
6 it was $0.025 \mathrm{~m}$. The target depth response time is close to $35 \mathrm{~s}$. The maximum control magnitude is around $8^{\circ}$.

\section{Case VII: BAUV control using internal model of two-fold exosystem: $\omega_{f}=8 \mathrm{~Hz}$, nominal parameters}

It is assumed that the fins are oscillating at $8 \mathrm{~Hz}$. For obtaining improved responses, a fifth-order servo compensator for the nominal values of the parameters is designed. Simulation results of the nominal BAUV are shown in Figure 8. 
We observe that target depth is attained and oscillations of fundamental and second harmonic in the tracking error are suppressed. Compared with the case of first-order servo compensator in Figure 6, we observe significant reduction in the peak magnitude of the steady-state tracking error. The magnitude of the tracking error is $0.004 \mathrm{~m}$ in Figure 8 which is substantially lesser than the tracking error magnitude of $0.025 \mathrm{~m}$ in Figure 6. The maximum control magnitude is around $30^{\circ}$ as in the case of Figure 4 . The target depth is reached in little over $30 \mathrm{~s}$.

\section{Case VIII: BAUV control using internal model of two-fold exosystem: $\omega_{f}=8 \mathrm{~Hz},-25 \%$ uncertainty}

To examine the robustness of the designed controller an uncertainty of $-25 \%$ is added to the nominal values of the vectors $f_{a}, f_{b}, m_{a}$ and $m_{b}$ for simulation. The selected responses are shown in Figure 9. The tracking error magnitude for the chosen uncertainty is around $0.002 \mathrm{~m}$. The maximum control magnitude and the target depth response time are similar in both Figures 8 and 9.

It is pointed out that although the controller is designed based on the linearised model, these results show robust performance even for $\pm 25 \%$ parametric uncertainties in the Fourier coefficients. These coefficients are used in the computation of the fin force and moment according to (3). It is noted that the input matrix (which is a function of the Fourier coefficients) plays a key role in shaping the responses. The robustness of the controller depends on the the weighting matrix $Q$ and scalar $r$ appearing in the performance index. Of course, there does not exist a simple way to determine their proper values. The values of $Q$ and $r$ have been selected here by observing the simulated responses in several trials.

\section{Conclusion}

Based on the non-linear servo regulation theory, a new design methodology for the control of multi-input BAUVs using pectoral-like oscillating fins was presented in this paper. For the dive plane control, a pair of pectoral-like harmonically heaving and pitching fins were used. The bias angle of pitch motion of the fin was treated as a control input. For the purpose of design, an exosystem of third order was introduced and the original time-varying non-linear system was embedded in a larger class of time-invariant non-linear system. For robust design, an internal model of $k$-fold exosystem was introduced. The augmented system, including the internal model, was stabilised using optimal control theory. In the closed-loop system, including the internal model of the $k$-fold exosystem, harmonic components of order up to $k$ of the tracking error are suppressed. This special property is not possible using averaging method or discretisation approach reported in literature. A simple servo compensator using only integral error feedback and a fifth-order servo compensator were designed. Simulation results were obtained, which showed robust depth control performance. Interestingly, the internal model of two-fold exosystem was capable of attenuating the depth tracking error to a negligible level in spite of uncertainties in the system parameters. It was seen that flexibility exists in the choice of weighting matrices for shaping responses using optimal control theory.

\section{References}

Azuma A. 1992. The bio-kinetics of flying and swimming. New York: Springer-Verlag.

Bandyopadhyay PR. 2005. Trends in biorobotic autonomous undersea vehicles. IEEE J Oceanic Eng. 30: 109-139.

Bandyopadhyay PR, Beal DN, Menozzi A. 2008. Biorobotic insights into how animals swim. J Exp Biol. 211: 206214.

Bandyopadhyay PR, Singh SN, Thivierge DP, Annaswamy AM, Leinhos HA, Fredette AR, Beal DN. 2008. Synchronization of animal-inspired multiple high-lift fins in an underwater vehicle using olivo-cerebellar dynamics. IEEE J Oceanic Eng. 33(4): 563-578.

Bizcurttas M, Dong H, Mittal R, Najjar F. 2005. Towards numerical simulation of flapping foils on fixed cartesian grids. Reno, NV: American Institute of Aeronautics and Astronautics. AIAA paper No. 2005-0079.

Chung S-J, Dorothy M, Stoner JR. 2009. Neurobiologically inspired control of engineered flapping flight. Paper No. AIAA 2009-1929, AIAA Infotech@Aerospace Conference; Seattle, WA.

Fish FE. 2004. Structure and mechanics of nonpiscine control surfaces. IEEE J Oceanic Eng. 29: 605-621.

Fossen TI. 1994. Guidance and control of oceanic vehicles. New York: Wiley Publications.

Huang J. 1995. Asymptotic tracking and disturbance rejection in uncertain nonlinear systems. IEEE Trans Automat Control. 40: $1118-1122$.

Huang J. 2004. Nonlinear output regulation: theory and applications. Philadelphia(PA): SIAM.

Kailath T. 1980. Linear systems. Englewood Cliffs (NJ): PrenticeHall.

Kato N. 2000. Performance in the horizontal plane of a fish robot with mechanical pectoral fins. IEEE J Oceanic Eng. 25(1): 121-129.

Kato N. 2002. Pectoral fin controllers. Neurotechnology for biometric robots. Cambridge (MA): MIT press. pp 325-350.

Kato N, Kamimura S. 2008. Bio-mechanisms of swimming and flying: fluid dynamics, biomimetic robots, and sports science. Berlin (Germany): Springer.

Lauder GV, Drucker EG. 2004. Morphology and experimental hydrodynamics of fish fin control surfaces. IEEE J Oceanic Eng. 29: 556-571.

Luca S. 1999. Analysis and control of flapping flight: from biological to robotic insects [doctor of philosophy dissertation]. [Berkeley(CA)]: University of California at Berkeley.

Meneveau C, Lund TS, Cabot WH. 1996. A Lagrangian dynamic subgrid-scale model of turbulence. J Fluid Mech. 319: 353385.

Naik MS and Singh SN. 2007. Oscillatory adaptive yaw-plane control of biorobotic autonomous underwater vehicles using pectoral-like fins. Appl Bionics Biomech. 4(4): 137147. 
Naik MS, Singh SN, Mittal R. 2009. Indirect adaptive output feedback control of a biorobotic AUV using pectoral-like mechanical fins. Bioinspir Biomim. 4(2): 1-11.

Najjar FM, Mittal R, Rampunggoon P, Khanna A. 2003. Simulations of complex flows and fluid-structure interaction problems on fixed Cartesian grids. ASME Paper No. FEDSM200345577, American Society Of Mechanical Engineers; New York.

Narasimhan M, Dong H, Mittal R, Singh SN. 2006. Optimal yaw regulation and trajectory control of biorobotic AUV using mechanical fins based on CFD parameterization. J Fluids Eng. 128: 687-698.

Seo K, Chung S-J, Slotine J-J E. 2008. CPG-based control of a turtle-like underwater vehicle. Proceedings of the Robotics: Science and Systems Conference IV; Zurich, Switzerland.

Sfakiotakis M, Lane DM, Davies JBC. 1999. Review of fish swimming modes for aquatic locomotion. IEEE J Oceanic Eng. 24(2): 237-253.

Singh SN, Simha A, Mittal R. 2004. Biorobotic AUV maneuvering by pectoral fins: inverse control design based on CFD parameterization. IEEE J Oceanic Eng. 29: 777785 .

Triantafyllou GS, Triantafyllou MS. 1995. An efficient swimming machine. Sci Amer. 272: 64-70.

Triantafyllou MS, Techet A, Hover F. 2003. Review of experimental work in biomimetic foils. 13th International Symposium on Unmanned Untethered Submersible Technology (UUST); New England Center, Durham, NH.

Triantafyllou MS, Techet A, Hover F. 2004. Review of experimental work in biomimetic foils. IEEE J Oceanic Eng. 29: 585-594.

Yamamoto I, Terada Y, Nagamatu T, Imaizumi Y. 1995. Propulsion system with flexible/rigid oscillating fin. IEEE J Oceanic Eng. 20(1): 23-30.
Udaykumar HS, Mittal R, Rampunggoon P, Khanna A. 2001. A sharp interface cartesian grid method for simulating flows with complex moving boundaries. J Comput Phys. 174: 345380 .

\section{Appendix}

Matrices $A_{p}^{[l]}$ and minimum polynomials

$$
\begin{aligned}
A_{p}^{[2]}= & {\left[\begin{array}{ccc}
0 & 2 \omega_{f} & 0 \\
-\omega_{f} & 0 & \omega_{f} \\
0 & -2 \omega_{f} & 0
\end{array}\right], } \\
A_{p}^{[3]}= & {\left[\begin{array}{cccc}
0 & 3 \omega_{f} & 0 & 0 \\
-\omega_{f} & 0 & 2 \omega_{f} & 0 \\
0 & -2 \omega_{f} & 0 & \omega_{f} \\
0 & 0 & -3 \omega_{f} & 0
\end{array}\right], } \\
A_{p}^{[4]}= & {\left[\begin{array}{ccccc}
0 & 4 \omega_{f} & 0 & 0 & 0 \\
-\omega_{f} & 0 & 3 \omega_{f} & 0 & 0 \\
0 & -2 \omega_{f} & 0 & 2 \omega_{f} & 0 \\
0 & 0 & -3 \omega_{f} & 0 & \omega_{f} \\
0 & 0 & 0 & -\omega_{f} & 0
\end{array}\right] . }
\end{aligned}
$$

Computing the minimum polynomials $p^{[1]}(\lambda)$ of $A^{[1]}$ and $p^{[k]}(\lambda)$ of $A_{p}^{[k]}(k=2,3,4)$, one finds that

$$
\begin{aligned}
& p^{[1]}=\lambda\left(\lambda^{2}+\omega_{f}^{2}\right), \\
& p^{[2]}=\lambda\left(\lambda^{2}+\left(2 \omega_{f}\right)^{2}\right), \\
& p^{[3]}=\left(\lambda^{2}+\omega_{f}^{2}\right)\left(\lambda^{2}+\left(3 \omega_{f}\right)^{2}\right), \\
& p^{[4]}=\lambda\left(\lambda^{2}+\left(2 \omega_{f}\right)^{2}\right)\left(\lambda^{2}+\left(4 \omega_{f}\right)^{2}\right) .
\end{aligned}
$$



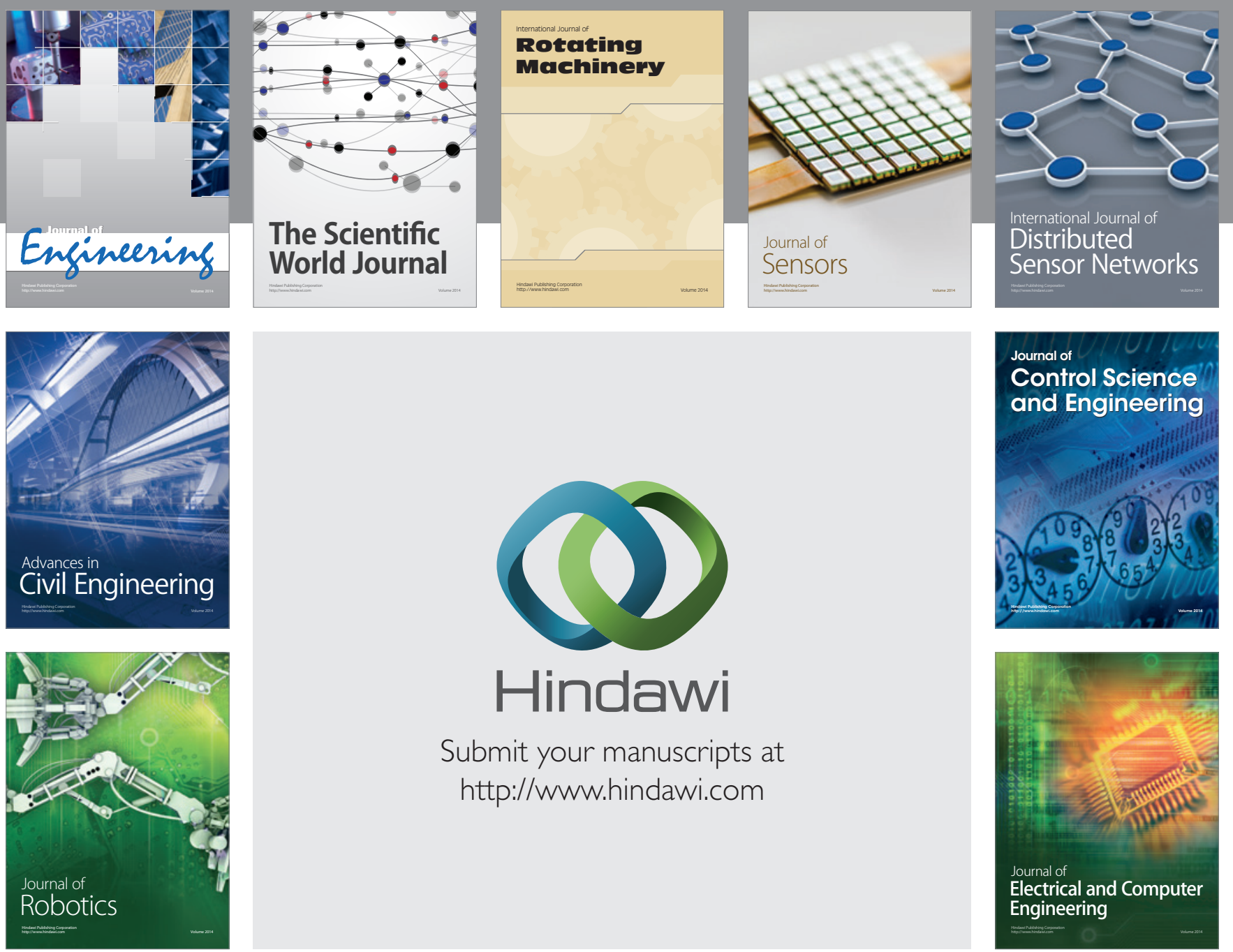

Submit your manuscripts at

http://www.hindawi.com
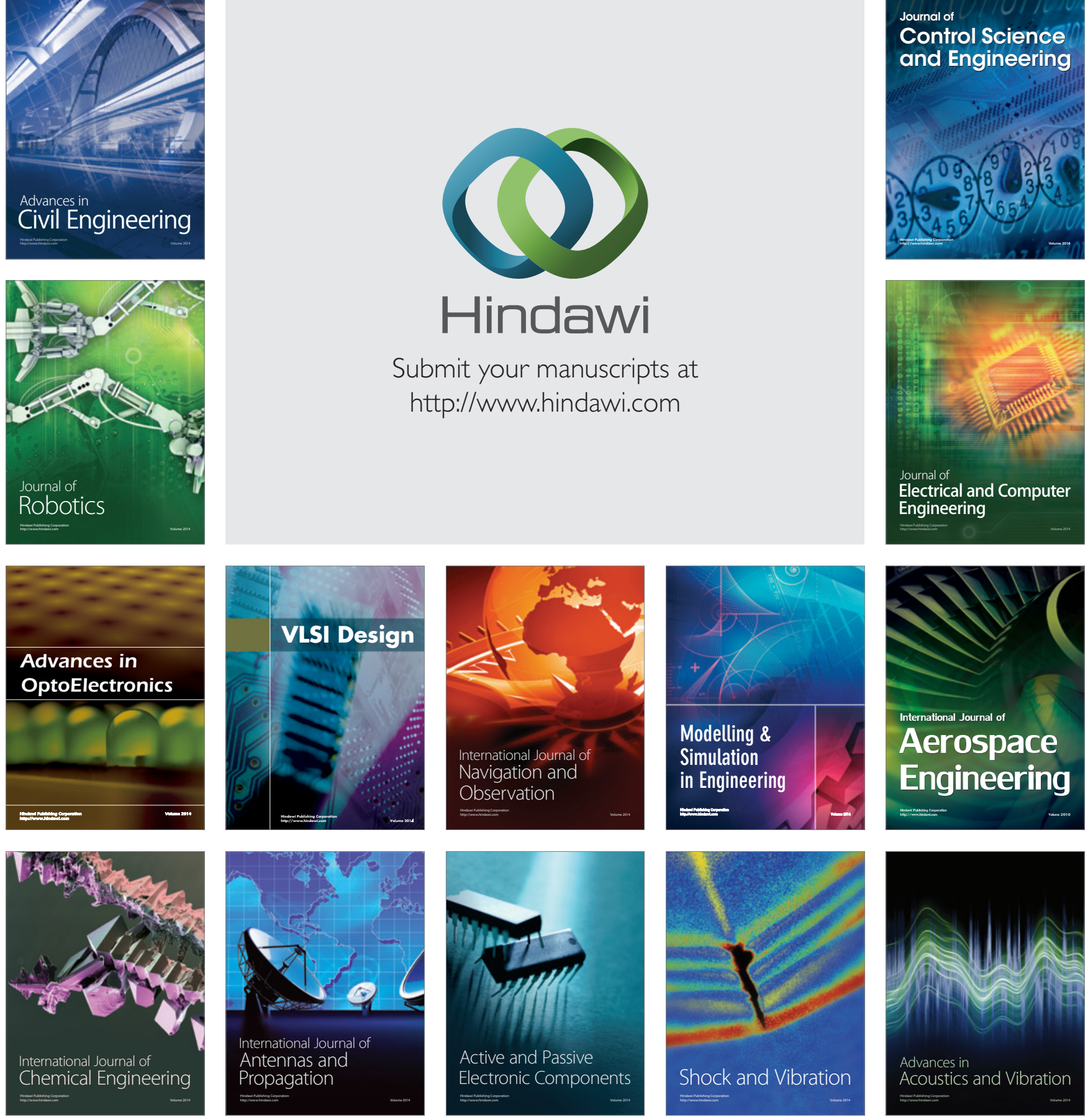